\title{
Spectral imaging maps of AR Lacertae
}

\section{Results from IUE observations in 1994 October}

\author{
I. Pagano ${ }^{1}$, M. Rodonò ${ }^{1,2}$, J. L. Linsky ${ }^{3}$, J. E. Neff ${ }^{4}$, F. M. Walter ${ }^{5}$, Zs. Kövári ${ }^{6}$, and L. D. Matthews ${ }^{7}$ \\ 1 Osservatorio Astrofisico di Catania, Via Santa Sofia 78, 95125 Catania, Italy \\ 2 Dipartimento di Fisica e Astronomia, Università di Catania, Via Santa Sofia 78, 95125 Catania, Italy \\ 3 JILA, University of Colorado and NIST, Boulder, CO 80309-0440, USA \\ 4 Department of Physics and Astronomy, College of Charleston, Charleston, SC 29424, USA \\ 5 Department of Physics and Astronomy, State University of New York, Stony Brook, NY 11794-3800, USA \\ ${ }^{6}$ Konkoly Observatory of the Hungarian Academy of Sciences, 1525 Budapest, Box 67, Hungary \\ 7 NRAO, Charlottesville, VA 22903, USA
}

Received 12 July 2000 / Accepted 6 October 2000

\begin{abstract}
In October 1994 the RS CVn eclipsing binary AR Lacertae was monitored by IUE during two orbital cycles. We have used 59 low resolution spectra, acquired in the range 1150-1950 $\AA$, to derive light curves for emission lines formed at different temperatures between about 10000 and $200000 \mathrm{~K}$ and to study the flare signatures versus temperature of line formation. We have analyzed a sequence of 59 high resolution Mg II $k$ line profiles using multi-Gaussian fits (the spectral imaging technique) to derive information of the spatial structure of the chromospheres of both stars in the AR Lac system. We discuss the quiet chromospheric emission from both stars, and we show that enhanced Mg II emission is present in extended structures corotating with the K0 IV star and close to the system center-of-mass. We also report on evidence for absorbing structures toward both the stars. This study of AR Lac used the new orbital elements of Marino et al. (1998) and spectra reduced and calibrated with NEWSIPS. A comparison between fluxes derived from the analyzed NEWSIPS spectra and from IUESIPS spectra is provided.
\end{abstract}

Key words. line: profiles - stars: activity - stars: binaries: general - stars: chromospheres - stars: AR Lac ultraviolet: stars

\section{Introduction}

In this paper we report on the chromospheric structure of AR Lacertae (=HD 210334), the brightest eclipsing RS CVn-type binary system. RS CVn systems are detached close binaries with two late type stars, one of spectral type late $\mathrm{F}$ or $\mathrm{G} \mathrm{V} / \mathrm{IV}$ and the other of approximate spectral type K0 IV/III. Due to the tidal coupling of the rotational and orbital periods that enforces higher-than-normal rotation, all features of stellar activity that depend on rotation period are consequently enhanced on these systems. The structures of the chromospheres, transition regions and coronae in RS CVn binaries have been derived by analyzing their UV, EUV, and X-ray spectra, the radio continuum emission and the few emission lines that lie in the visual region of their spectra.

AR Lacertae consists of a G2 IV primary and a K0 IV secondary star, which are spin-orbit coupled with

Send offprint requests to: I. Pagano, e-mail: ipa@sunct.ct.astro.it a 1.98 day period, and have a mass ratio $M_{1} / M_{2} \sim 0.89$ (Marino et al. 1998). The K star seems to be metaldeficient, but this is not the case for the G star (Naftilan \& Drake 1977). The physical characteristics and orbital elements of AR Lac are listed in Table 1. In this paper the individual $V$ magnitude values are computed using the luminosity ratio in the $V$ band found by Lanza et al. (1998), $L_{\mathrm{G}} / L_{\mathrm{K}}(V)=0.59$, and the $V$ magnitude of the system at light maximum given by Strassmeier et al. (1993), $V_{\max }=6.09$. The absolute magnitudes $M_{V}$ are then derived by adopting the distance $d=42 \mathrm{pc}$ measured by HIPPARCOS (Perryman et al. 1997). The radii are computed from the product of the mean fractional radii given by Lanza et al. (1998) $\left(r_{\mathrm{G}}=0.117\right.$, and $\left.r_{\mathrm{K}}=0.303\right)$ and the semi-major axis given by Marino et al. (1998) $\left(d_{\mathrm{G}-\mathrm{K}}=8.9 R_{\odot}\right.$ for orbital inclination $\left.i=87^{\circ}\right)$.

AR Lac was observed by the International Ultraviolet Explorer (IUE, see Boggess et al. 1978) several times since 1979. Maps of the chromospheres of the two stars were derived by Walter et al. (1987) and by Neff et al. (1989) 
Table 1. Physical parameters of AR Lacertae

\begin{tabular}{lrrll}
\hline & G2 IV & K0 IV & Unit & Reference \\
\hline$V$ & 7.17 & 6.59 & $\mathrm{mag}$ & present paper \\
$T_{\text {eff }}$ & 4820 & 5560 & $\mathrm{~K}$ & Lanza et al. (1998) \\
Mass & 1.13 & 1.26 & $M_{\odot}$ & Marino et al. (1998) \\
Radius & 1.48 & 2.69 & $R_{\odot}$ & present paper \\
$v_{\mathrm{e}} \sin i$ & 46 & 81 & $\mathrm{~km} \mathrm{~s}^{-1}$ & Strassmeier et al. (1993) \\
$K$ & 119.4 & 106.7 & $\mathrm{~km} \mathrm{~s}^{-1}$ & Marino et al. (1998) \\
$\gamma$ & -34.5 & $\mathrm{~km} \mathrm{~s}^{-1}$ & Marino et al. (1998) \\
$i$ & $87^{\circ}$ & & & Strassmeier et al. (1993) \\
$d$ & 42 & & pc & Perryman et al. (1997) \\
$M_{V}$ & 4.05 & 3.47 & $\mathrm{mag}$ & present paper \\
\hline
\end{tabular}

for two different epochs in 1983 and 1985, respectively. Preliminary results of successive IUE campaigns have been reported by Pagano et al. (1992a, 1992b, 1994, and 1995), and Kövári et al. (2000). We have embarked on a new study of AR Lac in the light of two recent improvements in the data: (a) new and more accurate orbital elements of AR Lac (Marino et al. 1998), which are a key factor when using the spectral imaging technique, as shown in Sect. 5.3, and (b) the release of the NEWSIPS (New Spectral Image Processing System) spectra, which provides data that are homogeneous in wavelength and flux calibrations. The main difference between the new orbital elements and those given by Sanford (1951), that have been used in all the analyses of IUE high resolution Mg II line profiles published to date, is the amplitude of the radial velocity curve of the K0 IV star. According to Marino et al. (1998) it is about $9 \mathrm{~km} \mathrm{~s}^{-1}$ less than previously assumed. In fact, the Sanford (1951) orbital elements lead to a discrepancy between the measured and predicted radial velocities of the K0 IV star, that are extremely evident close to quadratures (cf. Neff et al. 1989), and can mimic the effect that superficial inhomogeneities produce on the line profiles. Since, as we will show in Sect. 5.3, the information on the chromospheric spatial structure can be extracted from the line profiles only after the stellar radial velocities are assumed a priori, the accurate new orbital elements, as well as the accurate wavelength calibration provided by NEWSIPS are essential for our analysis. All the available IUE data of AR Lac can now be re-analyzed to obtain more accurate chromospheric maps and conclusive evaluations on the long-term variability of this system. Here we present the analysis of the IUE data set acquired in Oct. 1994, during the last of the IUE observing campaigns of this star covering more than one orbit. The analysis of the IUE data acquired in earlier observations will be the subject of future papers, as well as the study of the chromospheric variability on a time scale of about 15 years.

\section{The observations}

Ultraviolet observations of AR Lac were obtained by the IUE satellite over 5 days from Oct. 02 to Oct. 06 1994, with a continuous coverage of $\sim 102$ hours. The LWP camera obtained 59 high resolution spectra, and the SWP camera obtained 59 low resolution spectra. The large aperture was used for both the SWP and LWP observations. The Fine Error Sensor (FES) on board IUE was used to get a light curve in a wide optical band reducible through appropriate calibrations to the $V$ band. The typical sequence of observations consisted of repeated cycles of: FES, LWPHI, FES, SWP-LO. The SWP spectra exposure times were typically of $30 \mathrm{~min}$, while the LWP spectra were exposed generally for $60 \mathrm{~min}$. The data $\log$ is given in Tables 2 and 3. For each spectrum, the orbital/rotational phase at mid-exposure was computed by using the ephemeris $\mathrm{HJD}=2450692.5174, P=1.983188$ given by Marino et al. (1998), with the primary eclipse of the G2 IV star occuring at phase 0.0 .

At the time of our observations the FES was affected by scattered sunlight, the so called FES anomaly, which was superimposed to other diffuse light. The resulting FES light curve is therefore unreliable, and we will not discuss it further. The same source of the FES anomaly also affected the high resolution LWP spectra. In fact, between 2900 and $3000 \AA$, the solar spectrum is present as scattered light (Rodriguez Pascual 1993). SWP spectra were not affected by the FES anomaly.

\section{Data reduction and analysis method}

We have analyzed the Final Archive spectra retrived from the Multi-mission Archive at STScI (MAST), which were reduced by using the NEWSIPS (New Spectral Image Processing System) procedures. According to the IUE NEWSIPS Information Manual Ver. 2.0 (Garhart et al. 1997), the spectral resolution is about $0.17 \AA$ $\left(\sim 18 \mathrm{~km} \mathrm{~s}^{-1}\right)$ for the $\mathrm{Mg}$ II $h \& k$ region $(\lambda \sim 2800 \AA)$ in LWP high resolution spectra, and ranges from $\sim 3$ to $\sim 6.3 \AA$ in the SWP low resolution spectra between 1200 and $1900 \AA$ (750-1000 $\left.\mathrm{km} \mathrm{s}^{-1}\right)$. The instrumental profile is well described by a Gaussian function.

All spectral line measurements and analysis of the line profiles were done under IDL by means of the ICUR software package ${ }^{1}$.

We measured the SWP low-dispersion line fluxes by using Gaussian fits to the emission lines plus a quadratic

\footnotetext{
1 ICUR

(http://sbast3.ess.sunysb.edu/fwalter/ICUR/icur.html) is a general purpose screen-oriented data analysis program written in IDL for manipulating and analyzing one dimensional spectra. It is distributed to the public by the authors (F. M. Walter and J. E. Neff).
} 
Table 2. Data $\log$ of the IUE LWP high resolution observations made in 1994 October

\begin{tabular}{|c|c|c|c|c|c|c|c|}
\hline \multirow{2}{*}{$\begin{array}{r}\text { Image } \\
\text { LWP }\end{array}$} & \multirow{2}{*}{$\begin{array}{r}\text { Date } \\
\mathrm{dd}\end{array}$} & \multicolumn{3}{|c|}{ Start time } & \multirow{2}{*}{$\begin{array}{c}\text { Exp. } \\
\text { time } \\
\text { min }\end{array}$} & \multirow[t]{2}{*}{ Phase } & \multirow{2}{*}{$\begin{array}{c}\text { Panel } \\
\left({ }^{*}\right)\end{array}$} \\
\hline & & hh & $\min$ & $\mathrm{sec}$ & & & \\
\hline 29290 & 2 & 5 & 6 & 55 & 60 & 0.0965 & $\mathrm{a}$ \\
\hline 29291 & 2 & 7 & 14 & 24 & 60 & 0.1413 & b \\
\hline 29292 & 2 & 9 & 2 & 4 & 50 & 0.1774 & ** \\
\hline 29293 & 2 & 10 & 34 & 2 & 60 & 0.2114 & c \\
\hline 29294 & 2 & 12 & 17 & 15 & 60 & 0.2474 & d \\
\hline 29295 & 2 & 14 & 2 & 9 & 60 & 0.2842 & e \\
\hline 29296 & 2 & 15 & 44 & 21 & 60 & 0.3199 & $\mathrm{f}$ \\
\hline 29297 & 2 & 17 & 42 & 58 & 60 & 0.3612 & $\mathrm{~g}$ \\
\hline 29298 & 2 & 19 & 27 & 16 & 60 & 0.3980 & $\mathrm{~h}$ \\
\hline 29299 & 2 & 21 & 8 & 15 & 60 & 0.4334 & $\mathrm{i}$ \\
\hline 29300 & 2 & 22 & 49 & 38 & 60 & 0.4687 & $\mathrm{j}$ \\
\hline 29301 & 3 & 0 & 31 & 4 & 60 & 0.5045 & $\mathrm{k}$ \\
\hline 29302 & 3 & 2 & 12 & 39 & 60 & 0.5398 & 1 \\
\hline 29303 & 3 & 3 & 56 & 29 & 60 & 0.5762 & $\mathrm{~m}$ \\
\hline 29304 & 3 & 5 & 46 & 25 & 60 & 0.6148 & $\mathrm{n}$ \\
\hline 29305 & 3 & 7 & 25 & 28 & 60 & 0.6494 & o \\
\hline 29306 & 3 & 9 & 8 & 13 & 60 & 0.6855 & $\mathrm{p}$ \\
\hline 29307 & 3 & 10 & 50 & 48 & 60 & 0.7212 & $q$ \\
\hline 29308 & 3 & 12 & 33 & 46 & 60 & 0.7573 & $\mathrm{r}$ \\
\hline 29309 & 3 & 14 & 22 & 34 & 60 & 0.7954 & $\mathrm{~s}$ \\
\hline 29310 & 3 & 16 & 11 & 42 & 60 & 0.8336 & $\mathrm{t}$ \\
\hline 29311 & 3 & 17 & 54 & 35 & 60 & 0.8697 & $\mathrm{u}$ \\
\hline 29312 & 3 & 19 & 47 & 19 & 60 & 0.9092 & $\mathrm{v}$ \\
\hline 29313 & 3 & 21 & 31 & 18 & 60 & 0.9457 & $\mathrm{w}$ \\
\hline 29314 & 3 & 23 & 24 & 55 & 60 & 0.9852 & $\mathrm{x}$ \\
\hline 29315 & 4 & 0 & 59 & 43 & 60 & 0.0185 & $\mathrm{y}$ \\
\hline 29316 & 4 & 2 & 43 & 22 & 60 & 0.0549 & $\mathrm{z}$ \\
\hline 29317 & 4 & 4 & 27 & 54 & 60 & 0.0913 & $* *$ \\
\hline 29318 & 4 & 6 & 7 & 19 & 60 & 0.1263 & b \\
\hline 29319 & 4 & 7 & 47 & 57 & 60 & 0.1614 & $* * *$ \\
\hline 29320 & 4 & 9 & 27 & 41 & 20 & 0.1894 & $* * *$ \\
\hline 29321 & 4 & 10 & 52 & 19 & 60 & 0.2261 & $\mathrm{c}$ \\
\hline 29322 & 4 & 12 & 31 & 56 & 60 & 0.2608 & d \\
\hline 29323 & 4 & 14 & 31 & 26 & 60 & 0.3028 & $\mathrm{e}$ \\
\hline 29324 & 4 & 16 & 14 & 40 & 60 & 0.3389 & $\mathrm{f}$ \\
\hline 29325 & 4 & 17 & 57 & 37 & 60 & 0.3750 & $\mathrm{~g}$ \\
\hline 29326 & 4 & 19 & 44 & 57 & 98 & 0.4191 & $\mathrm{~h}$ \\
\hline 29327 & 4 & 22 & 11 & 4 & 60 & 0.4639 & $\mathrm{j}$ \\
\hline 29328 & 4 & 23 & 56 & 17 & 60 & 0.5007 & $\mathrm{k}$ \\
\hline 29329 & 5 & 1 & 40 & 26 & 60 & 0.5371 & 1 \\
\hline 29330 & 5 & 3 & 27 & 23 & 60 & 0.5745 & $\mathrm{~m}$ \\
\hline 29331 & 5 & 5 & 7 & 55 & 60 & 0.6096 & $\mathrm{n}$ \\
\hline 29332 & 5 & 6 & 47 & 39 & 60 & 0.6446 & o \\
\hline 29333 & 5 & 8 & 45 & 34 & 30 & 0.6806 & $* * *$ \\
\hline 29334 & 5 & 9 & 58 & 12 & 45 & 0.7088 & $q$ \\
\hline 29335 & 5 & 11 & 34 & 17 & 60 & 0.7451 & $\mathrm{r}$ \\
\hline 29336 & 5 & 13 & 27 & 47 & 60 & 0.7846 & $\mathrm{~s}$ \\
\hline 29337 & 5 & 15 & 12 & 27 & 60 & 0.8214 & $\mathrm{t}$ \\
\hline 29338 & 5 & 16 & 55 & 39 & 60 & 0.8575 & $\mathrm{u}$ \\
\hline 29339 & 5 & 18 & 42 & 57 & 70 & 0.8967 & $\mathrm{v}$ \\
\hline 29340 & 5 & 20 & 47 & 47 & 70 & 0.9405 & $\mathrm{w}$ \\
\hline 29341 & 5 & 23 & 3 & 48 & 90 & 0.9916 & $\mathrm{x}$ \\
\hline 29342 & 6 & 1 & 17 & 32 & 60 & 0.0333 & $\mathrm{y}$ \\
\hline 29343 & 6 & 3 & 0 & 9 & 60 & 0.0693 & $\mathrm{z}$ \\
\hline 29344 & 6 & 4 & 43 & 54 & 60 & 0.1054 & $\mathrm{a}$ \\
\hline 29345 & 6 & 6 & 26 & 31 & 60 & 0.1415 & $* * *$ \\
\hline 29346 & 6 & 8 & 19 & 50 & 30 & 0.1758 & $* * *$ \\
\hline 29347 & 6 & 9 & 30 & 11 & 21 & 0.1991 & $* * *$ \\
\hline 29348 & 6 & 10 & 38 & 32 & 55 & 0.2288 & $\mathrm{c}$ \\
\hline
\end{tabular}

(*) Panel of Fig. 2.

(**) Spectrum lost.

$(* * *)$ Spectrum disregarded because of poor $\mathrm{S} / \mathrm{N}$.
Table 3. Data log of the IUE SWP low resolution observations made in 1994 October

\begin{tabular}{|c|c|c|c|c|c|c|}
\hline \multirow{2}{*}{$\begin{array}{l}\text { Image } \\
\text { SWP }\end{array}$} & \multirow{2}{*}{$\begin{array}{r}\text { Date } \\
\mathrm{dd}\end{array}$} & \multicolumn{3}{|c|}{ Start time } & \multirow{2}{*}{$\begin{array}{l}\text { Exp. } \\
\text { time } \\
\text { min }\end{array}$} & \multirow[t]{2}{*}{ Phase } \\
\hline & & hh & $\min$ & $\mathrm{sec}$ & & \\
\hline 52280 & 2 & 6 & 20 & 10 & 35 & 0.1180 \\
\hline 52281 & 2 & 8 & 24 & 11 & 30 & 0.1606 \\
\hline 52282 & 2 & 9 & 57 & 7 & 30 & 0.1932 \\
\hline 52283 & 2 & 11 & 40 & 37 & 30 & 0.2292 \\
\hline 52284 & 2 & 13 & 24 & 43 & 30 & 0.2656 \\
\hline 52285 & 2 & 15 & 7 & 53 & 30 & 0.3017 \\
\hline 52286 & 2 & 16 & 50 & 37 & 30 & 0.3378 \\
\hline 52287 & 2 & 18 & 51 & 35 & 30 & 0.3801 \\
\hline 52288 & 2 & 20 & 32 & 52 & 30 & 0.4155 \\
\hline 52289 & 2 & 22 & 12 & 49 & 30 & 0.4505 \\
\hline 52290 & 2 & 23 & 54 & 39 & 30 & 0.4862 \\
\hline 52291 & 3 & 1 & 35 & 32 & 30 & 0.5216 \\
\hline 52292 & 3 & 3 & 19 & 13 & 30 & 0.5580 \\
\hline 52293 & 3 & 5 & 2 & 4 & 30 & 0.5941 \\
\hline 52294 & 3 & 6 & 51 & 10 & 30 & 0.6323 \\
\hline 52295 & 3 & 8 & 30 & 35 & 30 & 0.6669 \\
\hline 52296 & 3 & 10 & 16 & 10 & 30 & 0.7040 \\
\hline 52297 & 3 & 11 & 58 & 26 & 30 & 0.7398 \\
\hline 52298 & 3 & 13 & 40 & 42 & 30 & 0.7755 \\
\hline 52299 & 3 & 15 & 26 & 38 & 30 & 0.8126 \\
\hline 52300 & 3 & 17 & 17 & 55 & 30 & 0.8515 \\
\hline 52301 & 3 & 19 & 5 & 3 & 30 & 0.8893 \\
\hline 52302 & 3 & 20 & 53 & 17 & 30 & 0.9271 \\
\hline 52303 & 3 & 22 & 37 & 21 & 30 & 0.9635 \\
\hline 52304 & 4 & 0 & 21 & 6 & 30 & 0.9999 \\
\hline 52305 & 4 & 2 & 5 & 18 & 30 & 0.0363 \\
\hline 52306 & 4 & 3 & 50 & 21 & 30 & 0.0731 \\
\hline 52307 & 4 & 5 & 32 & 59 & 30 & 0.1088 \\
\hline 52308 & 4 & 7 & 12 & 59 & 30 & 0.1438 \\
\hline 52309 & 4 & 8 & 52 & 35 & 30 & 0.1789 \\
\hline 52310 & 4 & 10 & 17 & 0 & 30 & 0.2086 \\
\hline 52311 & 4 & 11 & 57 & 27 & 30 & 0.2436 \\
\hline 52312 & 4 & 13 & 41 & 11 & 30 & 0.2801 \\
\hline 52313 & 4 & 15 & 38 & 30 & 30 & 0.3210 \\
\hline 52314 & 4 & 17 & 21 & 56 & 30 & 0.3571 \\
\hline 52315 & 4 & 19 & 4 & 26 & 30 & 0.3932 \\
\hline 52316 & 4 & 21 & 29 & 43 & 30 & 0.4439 \\
\hline 52317 & 4 & 23 & 16 & 45 & 30 & 0.4814 \\
\hline 52318 & 5 & 1 & 2 & 41 & 30 & 0.5185 \\
\hline 52319 & 5 & 2 & 49 & 50 & 30 & 0.5560 \\
\hline 52320 & 5 & 4 & 33 & 24 & 30 & 0.5924 \\
\hline 52321 & 5 & 6 & 12 & 49 & 30 & 0.6271 \\
\hline 52322 & 5 & 7 & 52 & 50 & 30 & 0.6621 \\
\hline 52323 & 5 & 9 & 23 & 20 & 30 & 0.6939 \\
\hline 52324 & 5 & 10 & 50 & 10 & 30 & 0.7244 \\
\hline 52325 & 5 & 12 & 39 & 20 & 30 & 0.7626 \\
\hline 52326 & 5 & 14 & 33 & 59 & 30 & 0.8025 \\
\hline 52327 & 5 & 16 & 19 & 28 & 30 & 0.8396 \\
\hline 52328 & 5 & 18 & 6 & 54 & 30 & 0.8771 \\
\hline 52329 & 5 & 19 & 58 & 58 & 40 & 0.9180 \\
\hline 52330 & 5 & 22 & 3 & 53 & 47 & 0.9630 \\
\hline 52331 & 6 & 0 & 40 & 2 & 30 & 0.0150 \\
\hline 52332 & 6 & 2 & 23 & 32 & 30 & 0.0511 \\
\hline 52333 & 6 & 4 & 7 & 4 & 30 & 0.0875 \\
\hline 52334 & 6 & 5 & 49 & 18 & 30 & 0.1232 \\
\hline 52335 & 6 & 7 & 44 & 10 & 30 & 0.1635 \\
\hline 52336 & 6 & 8 & 54 & 55 & 30 & 0.1880 \\
\hline 52337 & 6 & 10 & 1 & 33 & 30 & 0.2115 \\
\hline 52338 & 6 & 11 & 39 & 33 & 60 & 0.2511 \\
\hline
\end{tabular}


fit to the background. This fitting procedure allows multiple Gaussians, therefore blended lines can be fitted accurately by constraining the line width to the instrumental resolution.

The principal aim of high-resolution LWP spectra was to study the strong $\mathrm{MgII} h(\lambda 2803.531 \AA)$ and $k(\lambda$ $2796.352 \AA)$ emission line cores. In practice, we have focused our analysis on the Mg II $k$ line, because this line is much better exposed than the Mg II $h$ line. We found that the flux ratio between the two lines is $f_{k} / f_{h}=1.32 \pm 0.02$. The high-resolution Mg II $k$ line profiles were analyzed using the procedure described by Walter et al. (1987) and by Neff et al. (1989). The method, called the spectral imaging technique, uses the Doppler information in high-resolution emission line profiles obtained at several rotational phases to generate a map of the brightness distribution over the surface of the star. The technique is based on observations that show that the rotationally broadened line profiles are generally observed to be distorted and highly variable from one phase to the next. This is because the typical line profile is the result of several components: the stars' uniformly-distributed emission (hereafter called the "quiet" emission), the interstellar medium (ISM) absorption, and the emission arising from discrete active regions and/or flares. The latter is variable from one spectrum to another because the active regions and flares are intrinsically variable and because their visibility is modulated by the stellar rotation. Therefore, we have fitted each observed MgII $k$ line profile with multi-Gaussian components to disentangle each of these contributions.

The adopted fitting procedure (in ICUR) is based on the Bevington's (1969) CURFIT algorithm. The choice of Gaussian functions to fit the $\mathrm{Mg}$ II $k$ line profile is fully justified for several reasons. In the absence of stellar rotation, the intrinsic Mg II $h \& k$ line profiles should have central reversals and the line widths would be given by the analog of the Wilson-Bappu relation (see Ayres 1980; Elgarøy et al. 1997). However, stellar rotation "fills-in" the central reversal and enhances the Doppler emission core (Bielicz et al. 1985). Also, IUE has a very limited dynamic range, so the exposure times chosen to expose the emission core optimally are too short to detect significant emission far in the (perhaps non-Gaussian) wings. More deeply exposed spectra, however, could reveal wings that have non-Gaussian shapes. Finally, the stellar line profile is convolved with the IUE instrumental response, which is close to Gaussian. The net result is an observed line profile that is effectively Gaussian. In the following sections, all derived line widths $W$ are those corrected for the instrumental broadening. We used a square correction, $W^{2}=W_{\text {obs }}^{2}-W_{\text {inst }}^{2}$, with $W_{\text {inst }}=18 \mathrm{~km} \mathrm{~s}^{-1}$ (Garhart et al. 1997).

\section{Results from the SWP low resolution spectra}

The most relevant lines in SWP low resolution spectra, in order of intensity, are: Ly $\alpha$ (1216 ̊), C IV $(1548+1551 \AA)$,

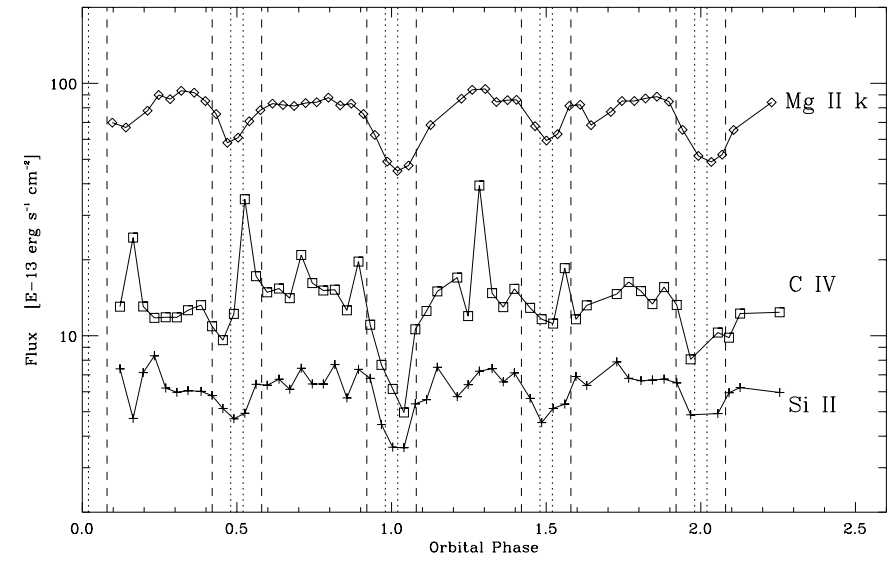

Fig. 1. AR Lacertae light curves in representative chromospheric ( $\mathrm{Mg}$ II $k$ and $\mathrm{Si}$ II) and transition region (C IV) lines. The dotted and dashed vertical lines show the durations of total (second to third contact) and partial eclipses

C II (1334+1336 ̊), Si II (1808+1817 ̊), He II (UV12 multiplet near $1640 \AA$ ), C I (multiplet at $1657 \AA$ ), C III (UV4 multiplet near $1176 \AA$ ), O I $(11302+1304+1306 \AA)$, and N v $(1239+1243 \AA)$. Because of the low detector sensitivity below the Ly-alpha line, in many spectra it was not possible to measure the $\mathrm{C}$ III line. Most of the spectra show a feature at $\sim 1668 \AA$ due to a blending of O III] (1666.1 $\AA$ ), Si I $(1666.4 / .6 \AA)$, and Al II (1670.8 $\AA$ ). Many spectra show a feature at $1355 \AA$ originating from a blending of the O I line at $1355.6 \AA$ with other minor O I and C I lines, plus possibly the Fe xxi line at $1354 \AA$. We also measured a weak feature at $\sim 1561 \AA$, with an integrated flux at Earth of $0.86 \pm 0.1110^{-13} \mathrm{erg} \mathrm{s}^{-1} \mathrm{~cm}^{-2}$, which is due to the C I multiplet at $1560 / 1 \AA$. Apart for the Ly $\alpha$, the integrated fluxes for the most prominent lines are listed in Table 4.

Figure 1 shows the AR Lac light curves in representative transition region (C IV) and chromospheric (Si II and $\mathrm{Mg}$ II $k$ ) lines. Both the chromospheric and transition region light curves show primary and secondary eclipses slightly wider than the eclipses of the photospheres. The mean fluxes, together with the mean out-of-eclipse fluxes and the mean quiet fluxes are given in Table 5. Three clear flare episodes are visible in the C IV light curve (exceeding by two times the out-of-eclipse standard deviation $\sigma$ ), plus possibly three other minor flare events $(\geq 1 \sigma)$. There is no clear signature of any of these flares in the chromospheric lines. It is worth noting that flares are visible in the $\mathrm{C}$ II line, whose temperature of line formation is $\sim 40000 \mathrm{~K}$, but not in the Si II line, which is mostly formed at $\sim 20000 \mathrm{~K}$. In Table 5 we list also the mean fluxes derived from the analysis of the same set of spectra but reduced by IUESIPS. We found that the NEWSIPS reduction systematically provides higher fluxes than IUESIPS, and the relative difference is generally larger for the low intensity lines. We have found only a slight improvement of the $\mathrm{S} / \mathrm{N}$ ratio measured in selected spectral intervals from IUESIPS to NEWSIPS for SWP low resolution spectra. 
Table 4. Fluxes at Earth in unit of $10^{-13} \mathrm{erg} \mathrm{s}^{-1} \mathrm{~cm}^{-2}$ of the emission lines measured in the low resolution SWP spectra (NEWSIPS reduction). See the text in Sect. 4 for line identification

\begin{tabular}{|c|c|c|c|c|c|c|c|c|c|c|c|c|}
\hline $\begin{array}{l}\text { SWP } \\
\text { Image }\end{array}$ & Phase & C III & $\mathrm{Nv}$ & O I & C II & $\mathrm{O}{ }{ }^{a}$ & Si IV & C IV & He II & $\mathrm{C}_{\mathrm{I}}$ & $\mathrm{O} \mathrm{III}]+{ }^{b}$ & Si II \\
\hline 52280 & 0.1179 & 5.02 & 2.37 & 3.74 & 6.08 & 1.15 & 3.50 & 13.04 & 5.51 & 3.23 & - & 7.40 \\
\hline 52281 & 0.1608 & 8.67 & 4.38 & 4.24 & 7.70 & 1.13 & 7.24 & 24.49 & 4.98 & 7.33 & 2.14 & 4.70 \\
\hline 52282 & 0.1933 & - & 2.30 & 3.37 & 8.24 & - & 2.82 & 13.06 & 6.16 & 5.85 & 0.97 & 7.15 \\
\hline 52283 & 0.2297 & 4.38 & 1.36 & 3.46 & 6.66 & 0.52 & 2.68 & 11.77 & 4.56 & 4.32 & 1.68 & 8.33 \\
\hline 52284 & 0.2661 & 4.28 & 2.62 & 3.78 & 6.70 & 1.04 & 3.99 & 11.84 & 4.67 & 3.80 & 1.66 & 6.21 \\
\hline 52285 & 0.3021 & 2.32 & 2.45 & 4.52 & 8.90 & - & 3.98 & 11.83 & 5.32 & 4.08 & - & 5.97 \\
\hline 52286 & 0.3380 & 4.46 & 2.21 & 4.51 & 8.28 & - & 5.07 & 12.63 & 7.38 & 4.00 & 1.75 & 6.06 \\
\hline 52287 & 0.3804 & 4.98 & 3.05 & 4.43 & 6.75 & 1.33 & 3.10 & 13.22 & 4.72 & 3.81 & - & 6.02 \\
\hline 52288 & 0.4158 & 4.34 & 2.82 & 3.26 & 6.69 & - & 3.50 & 10.90 & 4.84 & 4.34 & - & 5.79 \\
\hline 52289 & 0.4508 & 4.70 & 2.53 & 4.90 & 6.09 & 0.71 & 2.16 & 9.61 & 4.93 & 3.79 & - & 5.15 \\
\hline 52290 & 0.4862 & 0.25 & 2.55 & 3.25 & 5.50 & 1.12 & 2.66 & 12.21 & 6.14 & 3.96 & - & 4.69 \\
\hline 52291 & 0.5217 & 5.76 & 4.96 & 4.80 & 9.89 & 1.53 & 9.17 & 34.75 & 9.32 & 5.25 & - & 4.93 \\
\hline 52292 & 0.5581 & - & 3.13 & 6.21 & 10.70 & - & 5.27 & 17.22 & 7.01 & 4.12 & 1.22 & 6.42 \\
\hline 52293 & 0.5941 & 2.71 & 3.11 & 3.73 & 7.32 & - & 3.98 & 14.86 & 6.77 & 4.64 & 1.59 & 6.37 \\
\hline 52294 & 0.6325 & 3.56 & 3.32 & 2.86 & 7.51 & 0.70 & 4.70 & 15.39 & 6.37 & 4.74 & 2.01 & 6.73 \\
\hline 52295 & 0.6670 & 3.77 & 1.71 & 3.19 & 7.72 & - & 3.66 & 14.09 & 5.40 & 4.21 & 1.43 & 6.14 \\
\hline 52296 & 0.7039 & 4.86 & 3.74 & 4.60 & 8.35 & - & 6.09 & 20.89 & 7.40 & 4.51 & 1.14 & 7.43 \\
\hline 52297 & 0.7398 & 5.28 & 2.19 & 3.83 & 7.83 & 0.63 & 5.25 & 16.17 & 6.49 & 4.26 & 1.26 & 6.44 \\
\hline 52298 & 0.7758 & - & 2.95 & 4.35 & 8.86 & 1.18 & 4.64 & 15.11 & 6.63 & 4.18 & 1.69 & 6.44 \\
\hline 52299 & 0.8127 & 2.47 & 2.37 & 3.43 & 6.84 & - & 3.60 & 15.22 & 5.93 & 3.86 & 0.79 & 7.69 \\
\hline 52300 & 0.8516 & 2.74 & 2.18 & 4.07 & 6.66 & 0.72 & 3.85 & 12.62 & 5.87 & 4.22 & 0.70 & 5.67 \\
\hline 52301 & 0.8895 & 3.69 & 2.74 & 3.69 & 8.61 & 0.60 & 5.15 & 19.67 & 7.63 & 4.57 & - & 7.36 \\
\hline 52302 & 0.9270 & 4.02 & 2.52 & 2.87 & 5.73 & - & 2.42 & 11.05 & 6.17 & 3.74 & 0.78 & 6.78 \\
\hline 52303 & 0.9634 & 2.87 & 1.10 & 2.55 & 3.97 & - & 1.44 & 7.67 & 3.46 & 2.36 & - & 4.45 \\
\hline 52304 & 0.9998 & 4.25 & 1.45 & 1.81 & 2.61 & 1.42 & 2.47 & 6.16 & 3.06 & 1.91 & - & 3.62 \\
\hline 52305 & 0.0363 & 3.20 & 0.96 & 1.47 & 2.48 & 1.49 & 1.54 & 4.97 & 3.55 & 1.93 & 0.63 & 3.60 \\
\hline 52306 & 0.0732 & - & 3.77 & 3.98 & 5.75 & 0.49 & 2.12 & 10.61 & 6.53 & 3.73 & 0.91 & 5.37 \\
\hline 52307 & 0.1092 & 2.59 & 1.91 & 3.93 & 5.70 & 1.29 & 2.65 & 12.54 & 5.95 & 3.27 & 1.68 & 5.58 \\
\hline 52308 & 0.1441 & 8.48 & 2.59 & 4.44 & 6.56 & - & 2.98 & 14.99 & 8.62 & 6.32 & 1.42 & 7.50 \\
\hline 52310 & 0.2086 & 4.47 & 3.97 & 3.66 & 8.45 & - & 4.51 & 17.00 & 8.02 & 4.71 & 2.06 & 5.74 \\
\hline 52311 & 0.2436 & 2.50 & 3.13 & 3.24 & 7.63 & - & 4.13 & 11.97 & 7.66 & 4.65 & 1.04 & 6.40 \\
\hline 52312 & 0.2800 & - & 6.50 & 6.64 & 13.61 & - & 11.53 & 39.38 & 8.79 & 5.35 & 2.32 & 7.24 \\
\hline 52313 & 0.3214 & 5.62 & 2.01 & 5.47 & 7.81 & 0.93 & 3.00 & 14.75 & 6.28 & 3.30 & 1.46 & 7.41 \\
\hline 52314 & 0.3573 & 5.78 & 2.79 & 3.74 & 7.09 & - & 5.14 & 12.98 & 5.60 & 3.32 & 1.46 & 6.57 \\
\hline 52315 & 0.3933 & 3.50 & 4.65 & 4.15 & 7.48 & - & 5.55 & 15.34 & 6.37 & 3.87 & 4.71 & 7.13 \\
\hline 52316 & 0.4440 & 2.02 & 3.78 & 5.89 & 5.96 & 0.97 & 2.76 & 12.90 & 4.77 & 3.07 & - & 5.65 \\
\hline 52317 & 0.4819 & 3.25 & 3.06 & 2.95 & 6.01 & - & 5.10 & 11.63 & 6.00 & 3.83 & - & 4.53 \\
\hline 52318 & 0.5189 & 2.58 & 3.24 & 3.54 & 6.77 & - & 2.32 & 11.17 & 5.71 & 2.66 & 2.46 & 5.15 \\
\hline 52319 & 0.5563 & 4.72 & 2.40 & 3.54 & 7.55 & - & 4.30 & 18.53 & 6.03 & 6.68 & - & 5.36 \\
\hline 52320 & 0.5927 & 4.50 & 2.68 & 3.17 & 7.27 & 0.67 & 3.25 & 11.62 & 5.95 & 5.34 & - & 6.90 \\
\hline 52321 & 0.6272 & 4.31 & 3.89 & 4.06 & 7.33 & 3.23 & 4.24 & 13.20 & 6.85 & 3.73 & 2.10 & 6.35 \\
\hline 52324 & 0.7247 & 3.79 & 2.60 & 3.24 & 7.10 & - & 5.13 & 14.63 & 6.59 & 3.49 & 2.10 & 7.88 \\
\hline 52325 & 0.7626 & 3.91 & 2.44 & 3.61 & 7.14 & 1.33 & 3.83 & 16.32 & 7.15 & 4.15 & 1.25 & 6.78 \\
\hline 52326 & 0.8030 & 3.29 & 3.23 & 3.99 & 8.20 & - & 3.82 & 15.02 & 6.34 & 4.30 & 2.14 & 6.64 \\
\hline 52327 & 0.8399 & 3.37 & 1.57 & 3.97 & 7.96 & - & 2.34 & 13.35 & 7.14 & 4.60 & 0.73 & 6.67 \\
\hline 52328 & 0.8773 & - & 2.55 & 3.01 & 6.43 & - & 5.97 & 15.59 & 6.47 & 3.95 & 2.38 & 6.73 \\
\hline 52329 & 0.9182 & - & 4.23 & 3.33 & 5.92 & - & 3.32 & 13.24 & 5.88 & 2.45 & - & 6.51 \\
\hline 52330 & 0.9635 & - & 2.46 & 2.80 & 3.43 & - & 2.48 & 8.07 & 3.70 & 3.25 & 0.50 & 4.86 \\
\hline 52332 & 0.0512 & 1.90 & 4.28 & 5.88 & 4.44 & - & 2.84 & 10.30 & 5.25 & 3.13 & 0.79 & 4.92 \\
\hline 52333 & 0.0876 & 2.62 & 1.91 & 2.96 & 5.24 & - & 4.68 & 9.83 & 5.81 & 2.97 & 1.26 & 5.95 \\
\hline 52334 & 0.1235 & 2.90 & 3.06 & 4.12 & 5.75 & - & 2.94 & 12.24 & 4.52 & 3.29 & 0.92 & 6.24 \\
\hline 52338 & 0.2511 & 3.67 & 2.67 & 4.11 & 8.06 & - & 3.82 & 12.39 & 5.66 & 4.44 & 1.15 & 5.96 \\
\hline
\end{tabular}

${ }^{a}$ O I $(1355.6 \AA)+$ other minor C I and O I lines + Fe Xxi $(1354 \AA)$.

${ }^{b} \mathrm{O}$ III] $(1666.1 \AA)+$ Si I $(1666.4 / .6 \AA)+\mathrm{Al}$ II $(1670.8 \AA)$. 
Table 5. Mean fluxes at Earth in unit of $10^{-13} \mathrm{erg} \mathrm{s}^{-1} \mathrm{~cm}^{-2}$ (NEWSIPS data reduction)

\begin{tabular}{|c|c|c|c|c|c|c|c|c|c|c|c|c|}
\hline & Mg II $k$ & $\mathrm{C}_{\text {III }}$ & $\mathrm{Nv}$ & $\mathrm{O} \mathrm{I}$ & $\mathrm{C}_{\mathrm{II}}$ & $\mathrm{OI}+{ }^{a}$ & Si IV & C IV & He II & $\mathrm{CI}_{\mathrm{I}}$ & $\mathrm{O} \mathrm{III}]+{ }^{b}$ & Si II \\
\hline \multicolumn{13}{|c|}{ Out-of-eclipses spectra } \\
\hline Mean & 82.3 & 4.1 & 2.9 & 4.0 & 7.5 & 1.1 & 4.3 & 14.9 & 6.2 & 4.3 & 1.6 & 6.6 \\
\hline Error on the mean & 1.3 & 0.3 & 0.2 & 0.1 & 0.2 & 0.2 & 0.3 & 0.8 & 0.2 & 0.1 & 0.1 & 0.1 \\
\hline Standard deviation & 8.0 & 1.5 & 1.0 & 0.8 & 1.4 & 0.6 & 1.6 & 5.0 & 1.1 & 0.9 & 0.7 & 0.8 \\
\hline \multicolumn{13}{|c|}{ Out-of-eclipse spectra - no flaring phases } \\
\hline Mean & 82.0 & 3.9 & 2.7 & 3.9 & 7.2 & 1.1 & 3.9 & 13.6 & 6.1 & 4.1 & 1.6 & 6.6 \\
\hline Error on the mean & 1.3 & 0.2 & 0.1 & 0.1 & 0.2 & 0.2 & 0.2 & 0.3 & 0.2 & 0.1 & 0.1 & 0.1 \\
\hline Standard deviation & 7.3 & 1.3 & 0.7 & 0.7 & 0.9 & 0.7 & 0.9 & 1.7 & 1.0 & 0.7 & 0.8 & 0.7 \\
\hline \multicolumn{13}{|l|}{ All spectra } \\
\hline Mean & 75.5 & 3.9 & 2.8 & 3.8 & 7.0 & 1.1 & 4.0 & 14.2 & 6.0 & 4.0 & 1.5 & 6.1 \\
\hline Error on the mean & 1.9 & 0.2 & 0.1 & 0.1 & 0.3 & 0.1 & 0.2 & 0.8 & 0.2 & 0.1 & 0.1 & 0.1 \\
\hline Standard deviation & 13.6 & 1.5 & 1.0 & 1.0 & 1.8 & 0.6 & 1.8 & 5.7 & 1.3 & 1.1 & 0.8 & 1.0 \\
\hline \multicolumn{13}{|c|}{ All spectra from IUESIPS data reduction } \\
\hline Mean & 63.6 & 2.7 & 2.0 & 2.5 & 6.1 & - & 3.6 & 13.0 & 4.4 & 3.1 & 1.3 & 5.8 \\
\hline Error on the mean & 1.7 & 0.5 & 0.1 & 0.1 & 0.3 & - & 0.7 & 0.7 & 0.1 & 0.1 & 0.1 & 0.1 \\
\hline Standard deviation & 12.5 & 2.9 & 0.7 & 0.7 & 1.9 & - & 1.8 & 5.3 & 1.8 & 0.8 & 0.5 & 0.8 \\
\hline$\frac{\text { Flux }_{N}-\text { Flux }_{I}}{\text { Flux }_{I}}(c)$ & 0.19 & 0.44 & 0.44 & 0.55 & 0.15 & - & 0.12 & 0.09 & 0.37 & 0.32 & 0.19 & 0.05 \\
\hline
\end{tabular}

\footnotetext{
${ }^{a}$ O I $(1355.6 \AA)+$ other minor C I andO I lines + Fe XXI $(1354 \AA)$.

${ }^{b} \mathrm{O}$ III] $(1666.1 \AA)+\mathrm{Si}$ I $(1666.4 / .6 \AA)+\mathrm{Al}$ II $(1670.8 \AA)$.

${ }^{c} \mathrm{~N}=$ NEWSIPS, I = IUESIPS.
}

\section{Results from the LWP high resolution spectra}

\subsection{Mg // $k$ line profile variability}

In Fig. 2 we show the $51 \mathrm{Mg}$ II $k$ line profiles ${ }^{2}$ analyzed with the Spectral Imaging technique. The comparison of $\mathrm{Mg}$ II $k$ line profiles obtained at the same orbital phase (within 0.02 in phase) in successive orbits provides useful information on the intrinsic variability of the chromospheric emission. The spectra acquired close to the orbital quadratures show the emission of the two stars well separated; the major contribution comes from the K0 IV star that is located at longer wavelengths between phases 0 and 0.5 , and at shorter wavelengths between phases 0.5 and 1 . It appears that between phases 0.1 and 0.3 , the G2 IV star chromospheric emission (shorter wavelengths) was significantly less during the first observed orbit than during the second orbit. On the other hand, the emission of the K0 IV star chromosphere was enhanced between phases 0.25 and 0.40 during the first orbit with respect

\footnotetext{
${ }^{2}$ As indicated in Table 2, of the 59 acquired LWP spectra, 7 were not used in the Spectral Imaging analysis because of poor $\mathrm{S} / \mathrm{N}$ ratio, while 1 spectrum was lost. The spectra with low $\mathrm{S} / \mathrm{N}$ ratio were obtained during the high radiation US2 shifts, when both the signal was lower because exposures were shortened to avoid saturation, and the noise was increased, as was the background.
}

to the second one. Many of the spectra (cf. panels $d$ to $h$, $l, o, q, s$ to $w$, and $y$ ) show how dramatic the variations in line profiles can be from one orbit to the next.

\subsection{Three-Gaussian fits to the $\mathrm{Mg} / / \mathrm{k}$ line profiles}

Our first step was to fit all of the observed Mg II $k$ profiles by using two emission components (one for each star), one absorption component for the ISM, and a quadratic function for the background. We have chosen a quadratic function for the background because the Mg II $k$ emission line is superimposed over a broad absorption profile. We constrained only the FWHM of the absorption component to be equal to the instrumental width.

The interstellar line wavelength provides a constantvelocity reference on the spectrum. Thus, a check of the quality of the relative wavelength calibration among all the acquired spectra is given by comparing the measured wavelengths of the ISM absorption line. Since we find a standard deviation of the ISM line central wavelengths of $0.0687 \AA$, i.e. of the order of 1 bin (reciprocal dispersion $\sim 0.0674 \AA / \mathrm{bin}$ ), we can conclude that the relative wavelength calibration is quite good. Although Pagano (1993) found that IUESIPS spectra obtained close in time, e.g., during a typical 2-4 days observing run, have accurate relative wavelength scales, wavelength offsets were present when comparing AR Lac IUE data sets acquired in different epochs. The existence of such wavelength offsets 

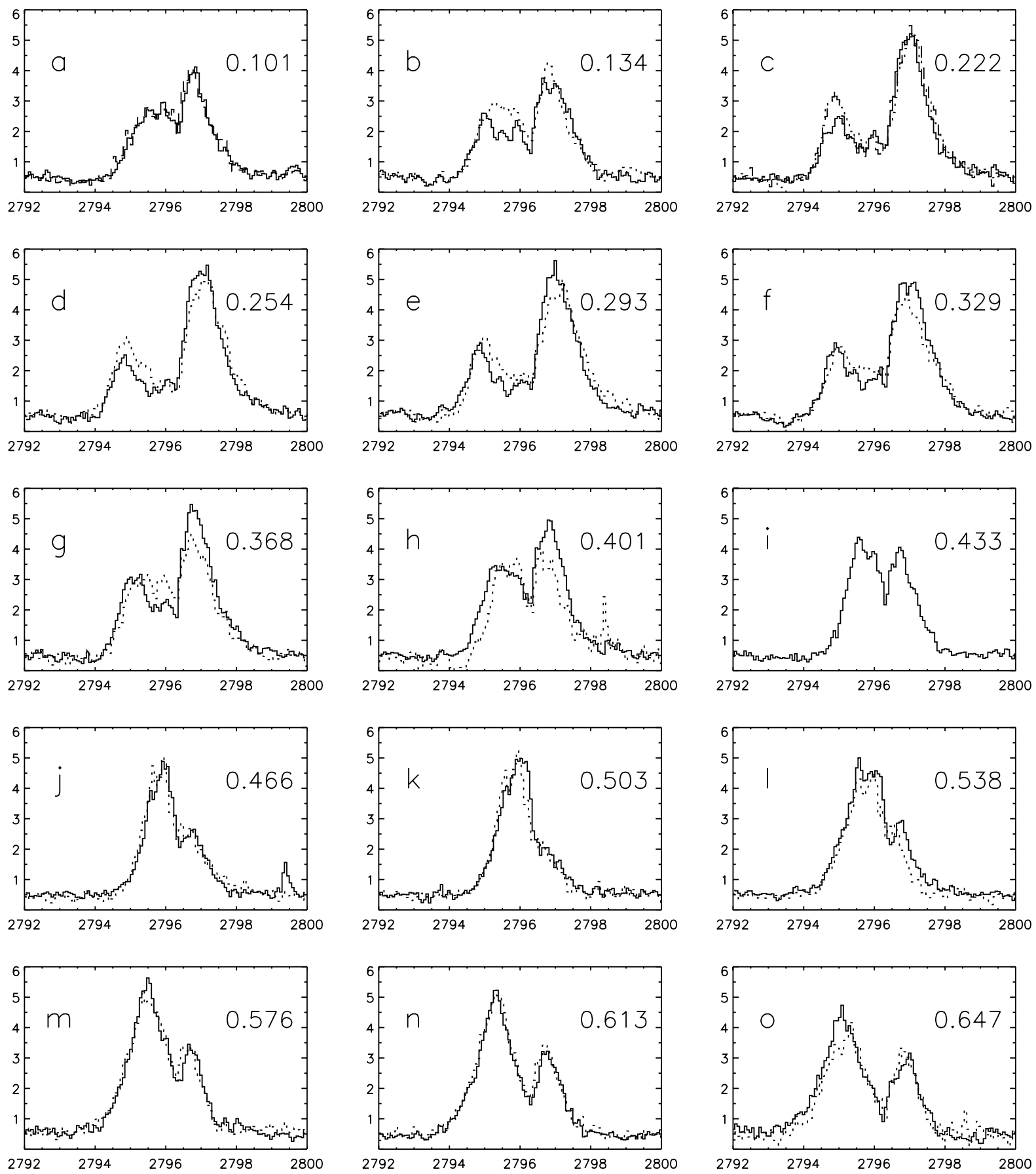

Fig. 2. a) The $51 \mathrm{Mg}$ II k line profiles analysed with the Spectral Imaging technique. Spectra acquired at orbital phases within 0.02 are plotted in the same panel, using a solid line for the 1st orbit, a dotted line for the 2nd orbit and a dashed line for the 3 th orbit (only panels $\mathbf{a}$, and $\mathbf{c}$ ). The mean phase is given in each panel. See Table 2 for the identification of the spectra of each panel. The $y$-axis units are fluxes in units of $10^{-12} \operatorname{erg~s}^{-1} \mathrm{~cm}^{-2} \AA^{-1}$, the $x$-axis are wavelength in $\AA$

among spectra acquired years apart, implies that the absolute wavelength calibration of IUESIPS spectra is not reliable (see also Thompson et al. 1990, for a discussion on the wavelength calibration problems present in IUESIPS data). Instead, the NEWSIPS data show very stable wavelengths even comparing spectra acquired in different observing runs. In fact, we have performed three-Gaussian fits, using the procedure explained above, to 221 out of the $240 \mathrm{LWP}(/ \mathrm{R})$ high resolution spectra of AR Lac acquired by IUE since 1979, discarding from our analysis only the poorly exposed spectra. We find that the ISM absorption line wavelengths are normally distributed with a mean of $2796.3425 \pm 0.0041$, and a standard deviation of 0.0602 , that is less than 1 bin. Therefore, according to Nichols \& Linsky (1996), and Barylak \& Ponz (1998), we find that the relative wavelength calibration performed 

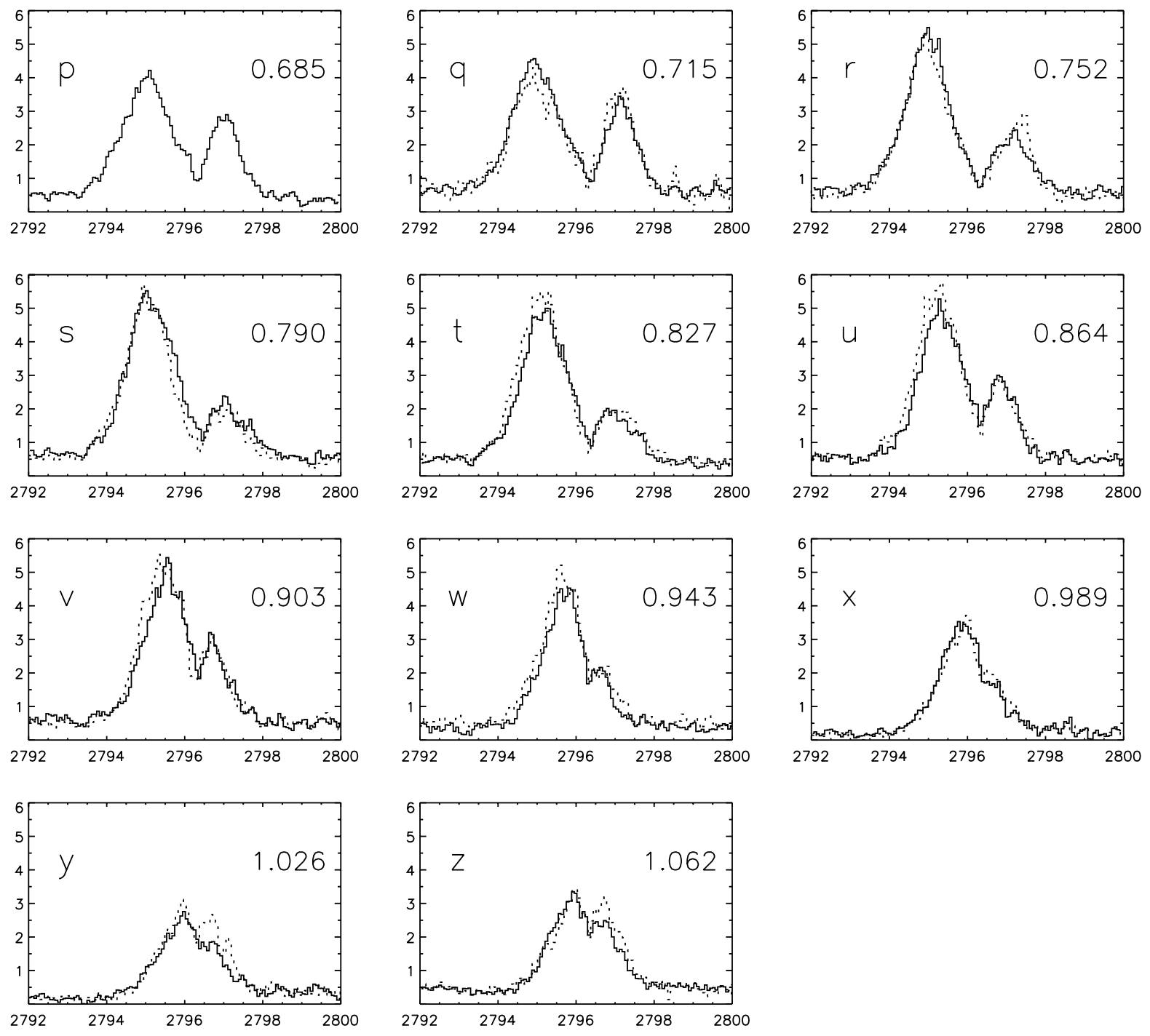

Fig. 2. b) See caption in Fig. 2a

by NEWSIPS is very accurate also comparing spectra acquired years apart. This fact allow us to be confident also on the absolute wavelength scale.

The mean central wavelength of the ISM absorption feature in the Mg II $k$ line toward AR Lac is 2796.3354 $0.0094 \AA\left(-1.8 \pm 1.0 \mathrm{~km} \mathrm{~s}^{-1}\right)$ according to the analysis of the 1994 data set. This value was best refined to $-1.0 \pm 0.4 \mathrm{~km} \mathrm{~s}^{-1}$ (heliospheric) by analyzing 221 of the $240 \mathrm{LWP}(/ \mathrm{R})$ high resolution spectra of AR Lac acquired by IUE since 1979 .

As shown in Fig. 3, the stellar radial velocities measured from the central positions of the Gaussians resulting from the three component fits are very close to those expected from the orbital elements derived by Marino et al. (1998). Therefore, the observed Mg II $k$ line profiles can be reasonably reproduced by our simple model (two emission and one absorption component). However, the integrated fluxes and especially the line widths show abrupt changes with orbital phase (cf. Fig. 4) that cannot be consistent with spatially uniform and static chromospheres.
Our next step was to separate the spatially uniform and static chromospheres (the "quiet" emission) from the active region and flare emission.

The results we have obtained from the 3-Gaussian fits suggest that a constant function is appropriate enough to fit the continuum in a region very close to the line emission core.

\subsection{Multi-Gaussian fits to the $\mathrm{Mg} / / \mathrm{k}$ line profiles}

We based our analysis on a criterion that distinguishes between the emission due to the two quiet stellar chromospheres and the emission arising from discrete bright regions. While the former kind of emission is constant during the orbit, except during the transit and occultation phases, the latter type of emission varies from one spectrum to the next either because the visibility of the active regions is modulated by stellar rotation (as in the case of a chromospheric active region) or because the flux arising from those regions that are intrinsically variable 


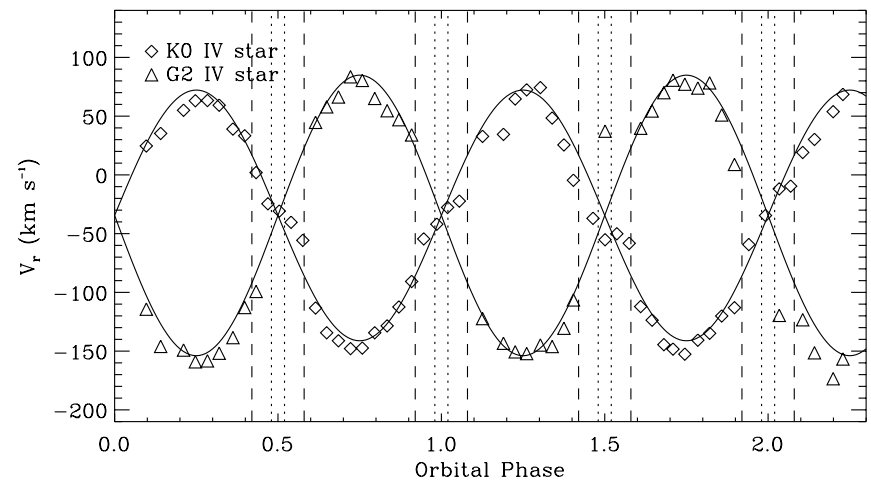

Fig. 3. Radial velocities of the K0 IV and G2 IV stellar emission components resulting from a free 3-Gaussian fit (two emission and one absorption components) to the $\mathrm{Mg}$ II $k$ line profiles. The solid lines are the stellar radial velocities predicted with the orbital elements of Marino et al. (1998)

(as in the case of a flares). Thus, the primary criterion that we used to determine the best solution of a multi-Gaussian fit to each line profile from among the many possible solutions was that the width and flux of the Gaussians that fit the quiet emission from the two stars vary with phase as smoothly as possible. To satisfy this condition additional components to the three used in the previous step were required to fit the observed $\mathrm{Mg}$ II $k$ line profiles. To reduce the number of free parameters of the fit we $a$ ) used a maximum of five Gaussians, b) froze the central wavelengths of the two components that fit the stellar quiet emission to the values predicted by the orbital elements, c) constrained the width of the interstellar medium absorption line to the instrumental width, and $d$ ) fit the background with a constant instead of a quadratic function. In fact, from the previous step we had already concluded that this choice is adequate in the spectral region very close to the line core. We used the orbital elements recently derived by Marino et al. (1998), and for the spectra acquired during eclipses we computed the equivalent radial velocities of the visible part of the stars. In Fig. 5 we show two examples of five-Gaussian fits to typical line profiles.

For the data we are analyzing here, the major advantage provided by the use of NEWSIPS instead of IUESIPS release is the reliability of the wavelength calibration in the NEWSIPS case (cf. Sect. 5.2). In fact, we can constrain the centroids of the Gaussians which fit the stellar quiet emission to the stellar radial velocities thanks to the accurate wavelength calibration provided by NEWSIPS. Instead, the substantial improvement in $\mathrm{S} / \mathrm{N}$ from IUESIPS to NEWSIPS in the spectral region containing the Mg II lines does not imply significant variations in the results of the multi-Gaussian fit to the $\mathrm{Mg}$ II $k$ lines.

We interpreted the additional components as active regions or another type of structure according to the behaviour of their radial velocity along the orbit. Moreover, the coverage of two sequential orbits allowed us to look for repeated detections, that are suggestive of emission arising in a long-lived active region or in a flaring site.

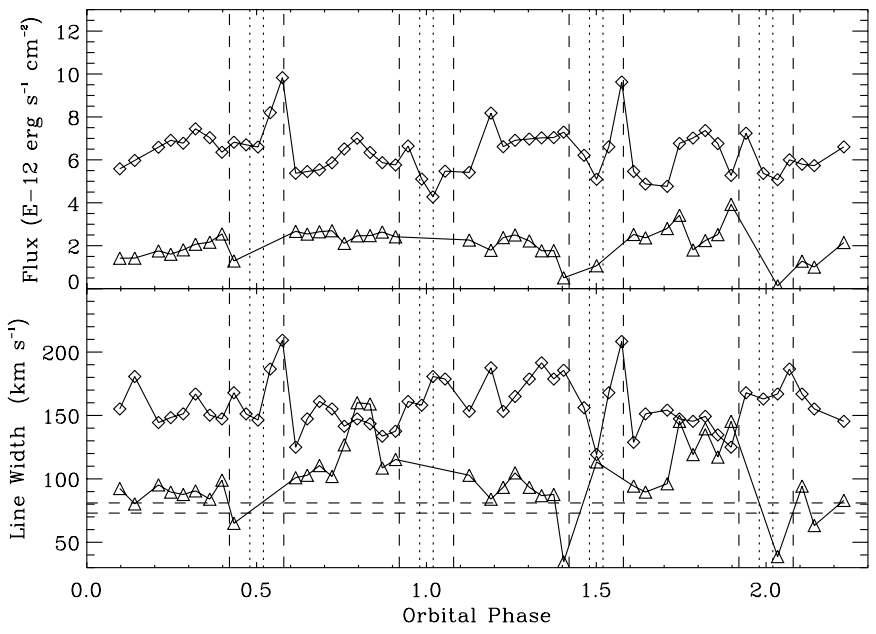

Fig. 4. Integrated fluxes and line widths of the K0 IV ( $d i$ amonds) and G2 IV (triangles) stellar emission components resulting from a free 3-Gaussian fit (two emission and one absorption components) to the line profiles. In the bottom panel the horizontal dashed-lines are drawn at the Wilson-Bappu line widths of the stars ( 81 and $73 \mathrm{~km} \mathrm{~s}^{-1}$ for the $\mathrm{K}$ and $\mathrm{G}$ stars, respectively), according to the Özeren et al. (1999) relation
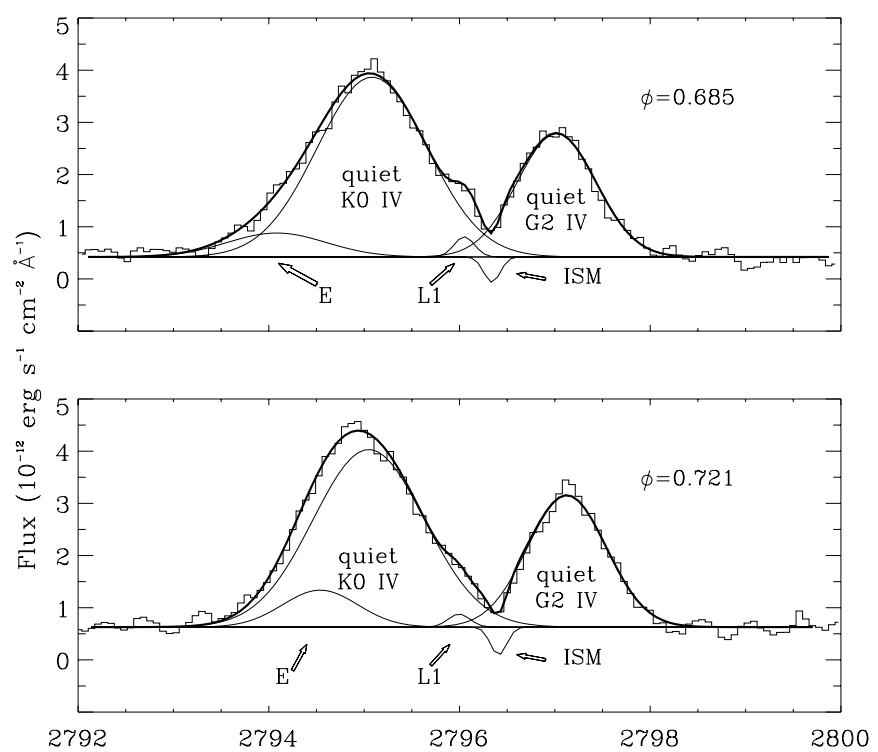

Fig. 5. Two examples of 5-Gaussian fits to typical Mg II $k$ line profiles. Each profile is best fit by the sum (solid thick line) of the quiet stellar emission components, the absorption component due to the interstellar medium (ISM), and two discrete emission components (E and L1). The E component is representative of those features, identified as active regions, which migrate across the line profile because of the stellar rotation. On the contrary, the radial velocity of the L1 component was almost constant, and the L1 feature was interpreted as due to emission near to the system center of mass

We have separated the quiet chromospheric emission from the two stars and identified discrete components of emission in all of the analyzed Mg II $k$ line profiles. Most of these features can be interpreted as signatures of active regions on the K0 IV star. However, we also found evidence 
for a bright $\mathrm{Mg}$ II region close to the Lagrangian point L1, and of an absorbing structure toward the G2 IV star.

\subsubsection{The quiet chromospheric emission from the two stars}

In Fig. 6 the widths and fluxes of the quiet emission from the two stellar chromospheres obtained from multiGaussian fits are shown versus orbital phase. The total flux from the system, i.e. the measured flux at Earth corrected for the interstellar absorption, is also shown in Fig. 6. As described in Sect. 5.3, we choose as best solutions of the multi-Gaussian fit to each line profile those that render the fluxes and line widths smoothly varying along the orbit. The best natural solutions with this approach are when the quiet fluxes out-of-eclipse are constant with phase. And in fact, this was what we find for the G2 IV star, which, outside of primary eclipse, is nearly constant at its mean value ${ }^{3}$ of $2.27 \pm 0.04(0.26)$ $10^{-12} \mathrm{erg} \mathrm{s}^{-1} \mathrm{~cm}^{-2}$. While we were able to isolate quite well the quiet emission of the $\mathrm{G}$ star, the same was not possible for the $\mathrm{K}$ star. In fact, the Gaussian component representing the quiet chromospheric emission from the K0 IV star is variable, with a minimum at the primary eclipse and maxima close to the quadratures. For example, during the first observed primary eclipse, the K0 IV star flux is $\sim 33 \%$ less than the mean out-of-eclipse flux $\left[4.85 \pm 0.12(0.77) 10^{-12} \mathrm{erg} \mathrm{s}^{-1} \mathrm{~cm}^{-2}\right]$. Some of the observed K0 IV star variability may be due to the ellipsoidal effect, which predicts maximum flux at quadratures when the maximum apparent surface is visible to the observer. However, a $33 \%$ variation due to ellipsoidal effect would be visible also in a much deeper secondary eclipse. Instead, the K0 IV star flux measured during secondary eclipse is consistent with what we expect from the not eclipsed annular surface of a uniformly bright chromosphere whose flux is the mean out-of-eclipse flux given above. Moreover, assuming the ellipsoid semi-axes given by Lanza et al. (1998) and no limb effects, the ellipsoidal effect can be responsible of a relative flux difference not much greater than $\sim 8 \%$. Therefore, a $33 \%$ decrease of emission at the primary eclipse implies that the K0 IV star hemisphere opposite to the G2 IV star has an intrisically weaker chromospheric emission.

The fact that the Gaussian component describing the quiet emission from the K0 IV star is not constant means that the K0 IV star chromosphere is bright and inhomogeneous, but our procedure allows us to resolve only the major asymmetrical and rather compact structures. Of course this is imposed from our choice to limit to five

\footnotetext{
${ }^{3}$ Here and hereafter we report the error on the mean and in parentheses the $\pm 1 \sigma$ scatter around the mean. We do not include the contribution of a possible systematic error in the absolute flux scale. As for the absolute flux calibration of IUE high resolution spectra, Cassatella et al. (2000) have shown that its internal accuracy is about $4 \%$ and that it applies both to continuum and emission lines.
}

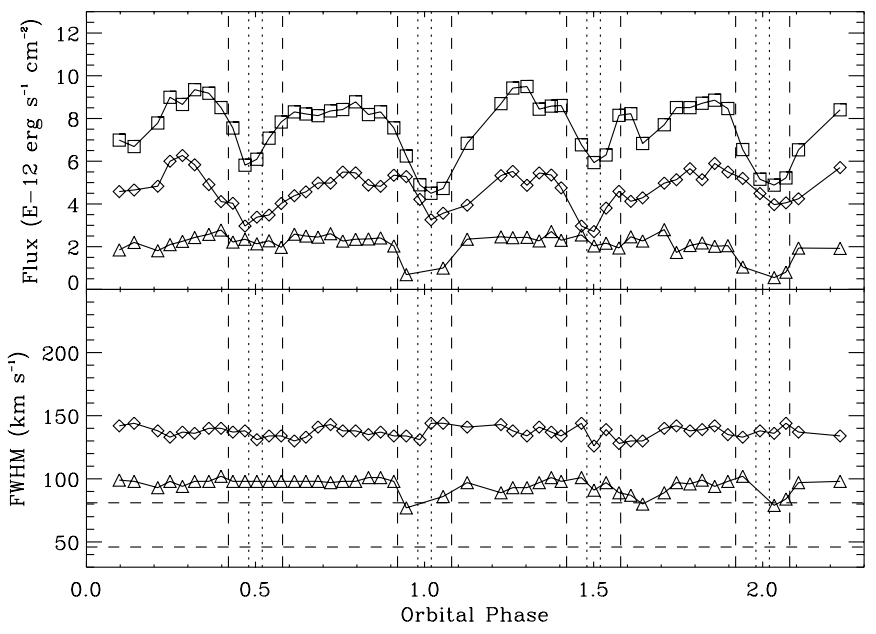

Fig. 6. Integrated fluxes and line widths of the K0 IV (diamonds) and G2 IV (triangles) quiet emission components, from a multi-Gaussian fit to the line profiles. In the top panel the Mg II $k$ total flux (i.e. the flux from the two quiet stars plus the flux from the other discrete emission and/or absorption components) is also plotted (squares). In the bottom panel the horizontal dashed-lines are drawn at the Wilson-Bappu line widths of the stars ( 81 and $73 \mathrm{~km} \mathrm{~s}^{-1}$ for the $\mathrm{K}$ and $\mathrm{G}$ stars, respectively), according to the Özeren et al. (1999) relation

the components of the multi-Gaussian fits to reduce the number of free parameters in the fits.

In analogy to what is seen from the C IV transition region light curve in Sect. 4, the Mg II light curve (total flux) also shows that the eclipses are wider than expected from the geometrical eclipse of the photospheres. The high resolution $\mathrm{Mg}$ II line spectra allow us to ascertain that the eclipses of the K0 IV star, but not of the G2 IV star, are wider than expected.

In Fig. 6 we also show the widths of the Gaussian components that fit the quiet chromospheric stellar emission, derived from the measured FWHM and corrected for the instrumental broadening as shown in Sect. 3. The measured line widths of the K0 IV star are normally distributed with a standard deviation $\sigma=4.4 \mathrm{~km} \mathrm{~s}^{-1}$ around a mean of $140.5 \pm 0.6 \mathrm{~km} \mathrm{~s}^{-1}$. The mean line width of the G2 IV star is $101.2 \pm 0.6 \mathrm{~km} \mathrm{~s}^{-1}\left(\sigma=4.1 \mathrm{~km} \mathrm{~s}^{-1}\right)$. The measured line widths can be compared with the values predicted by the analog of the Wilson-Bappu relation for the Mg II lines. Using the Özeren et al. (1999) relation derived for a sample of RS CVn stars:

$M_{V}=-12.01( \pm 0.89) \log W_{\mathrm{MgII} k}+26.41( \pm 1.82)$

the predicted values of $W_{\mathrm{MgII} k}$ are 81 and $73 \mathrm{~km} \mathrm{~s}^{-1}$ for the $\mathrm{K}$ and $\mathrm{G}$ stars, respectively. Therefore, the measured line widths are 1.7 (K star) and 1.3 (G star) times greater than predicted. Özeren et al. (1999) showed that the more active stars within a luminosity class have larger $\mathrm{Mg}$ II line widths, and there exists a qualitative dependence of the line width on the temperature minimum position - i.e. a deeper temperature minimum generally correlates with 
wider lines, and larger column mass at the temperature minimum correlates with wider line width.

\subsubsection{A bright $\mathrm{Mg}$ II emission region close to the Lagrangian point $\mathrm{L} 1$}

In 18 spectra, mostly between phases 0 and 0.5 , we have observed an emission feature at a wavelength that was almost constant with the orbital phase. The behaviour of the radial velocity of this feature versus phase is plotted in the bottom-left panel of Fig. 8 in the center of mass (Lagrangian point L1) rest frame. The mean velocity shift with respect to $\mathrm{L} 1$ is $-17 \pm 3 \mathrm{~km} \mathrm{~s}^{-1}$. The feature was observed during both orbital cycles, generally not resolved, with a mean flux of $0.21 \pm 0.0310^{-12} \mathrm{erg} \mathrm{s}^{-1} \mathrm{~cm}^{-2}$, i.e. $\sim 3 \%$ of the mean emission from the whole system. One possible interpretation for such a feature is that it is the signature of a hot spot due to mass exchange between the two stars. Evidence for a similar intersystem structure was found for the RS CVn-type binary system HR 1099 by Busà et al. (1999) and, independently, by Richards \& Rosolowsky (1998).

\subsubsection{An obscuring prominence close to the G2 IV star}

In 9 spectra we found evidence for an absorption feature that is responsible for an abrupt and dramatic weakness of the G2 IV star Mg II emission flux. In Fig. 7 we show as example two of these spectra. The radial velocities versus phase of this absorption feature, shown in the bottom-right panel of Fig. 8 in the G2 IV star rest frame, follow a normal distribution around a mean value of $4.5 \pm 6.5 \mathrm{~km} \mathrm{~s}^{-1}$ (reduced $\chi^{2}=0.54$ ). The behaviour can be also fit by a sinusoidal rotation curve of amplitude $30 \pm 13 \mathrm{kms}^{-1}$ (reduced $\chi^{2}=0.60$ ). Therefore, this feature can be explained by some obscuring material corotating with the G2 IV star at a latitude of about 50 degrees or higher, if it is located high in chromosphere, and centered at longitude $\sim 290$ degrees. The feature was resolved when detected close to phase 0.8 , otherwise it was generally unresolved. Also the absorbed flux is larger when the feature is observed closer to phase 0.8 than at other phases. The mean flux absorbed by this feature is $0.39 \pm 0.0610^{-12} \mathrm{erg} \mathrm{s}^{-1} \mathrm{~cm}^{-2}$, that is about $20 \%$ of the total flux of the G2 IV star.

\subsubsection{Active regions on the KO IV star}

The radial velocities of all of the remaining additional emission components found by multi-Gaussian fits to the line profiles are plotted in the rest frame of the K0 IV star versus phase in the five panels from the top of Fig. 8. The cross symbol in Fig. 8 represents the radial velocity of the one absorption feature in front of the K0 IV star, which was clearly detected only once at phase 0.7 during the 2nd orbit. Most of the discrete emission features appear to cross the K0 IV line profile from the blue to the red wing.
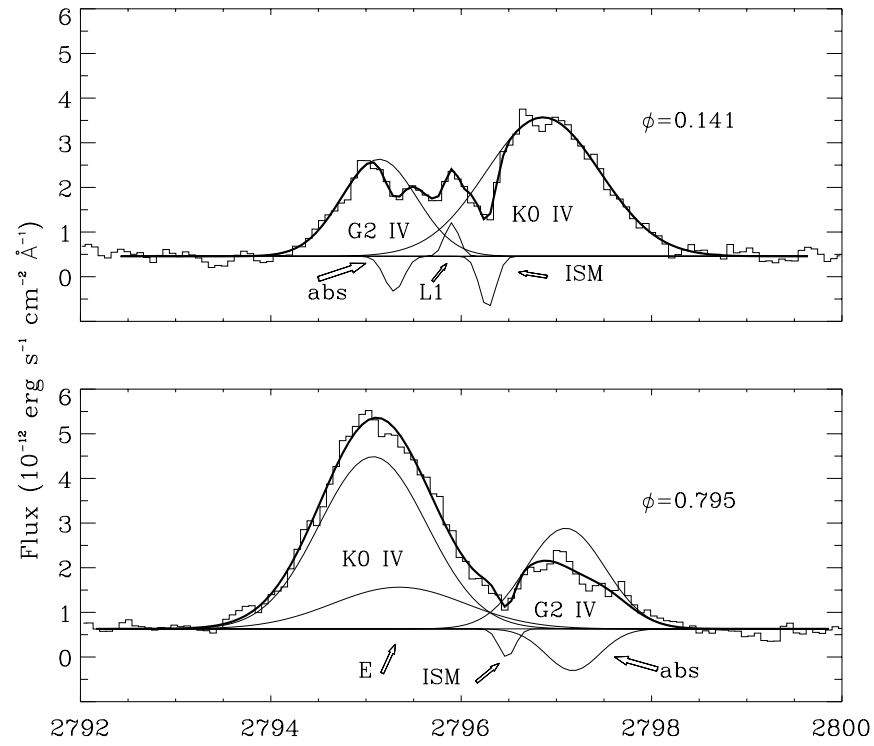

Fig. 7. Two of the nine MgII $k$ line profiles in which an absorption feature toward the G2 IV star was found

The behaviour of the radial velocities of these features versus orbital phase, cf. Fig. 8, can be represented by 5 sinusoidal curves, which we interpret as the radial velocity curves of 5 active regions corotating with the K0 IV star. From the parameters of the sinusoidal rotation curves, the active region longitude, latitude, and/or height above the K0 IV star photosphere can be derived. Neff et al. (1989) gave a complete description of the method used to infer the active region parameters and discussed the potential source of errors. Briefly, the longitude $L$ is given by the orbital phase at which the active region crosses the central meridian, which can be determined from the phase of zero velocity in the best fit radial velocity curve. The lower limit of the latitude, $l_{\min }$, can be derived only when the amplitude of the radial velocity curve is less than or equal to the stellar $v_{\mathrm{e}} \sin i$. Since the amplitudes $A$ of the active region radial velocity curves exceed the stellar rotational velocities $\left(v_{\mathrm{e}} \sin i\right)$, we write the amplitude as:

$A=v_{\mathrm{e}} \sin i H \cos l$,

where $H$ is the height above the photosphere in units of the stellar radius. Therefore, when $A \leq v_{\mathrm{e}} \sin i, l_{\text {min }}$ can be computed assuming $H=1$. Otherwise, the minimum height above the stellar photosphere, $H_{\text {min }}$, can be derived assuming $l=0$. An upper limit to the active region extention in longitude is given by the minimum line width measured for that active region, assuming that all of the deconvolved component width is due to velocity smearing from a resolved region on the star. This size is an upper limit, because there could be a substantial intrinsic width (i.e., turbulence and flows) and the active regions may not be homogeneous. There is no way to determine the extent of the active regions in latitude, since the inclination of AR Lac is $\sim 90^{\circ}$. Therefore, we assume that the active regions are circular. Finally, we compute the active region filling factors $(\mu)$ as the fraction of the stellar surface 

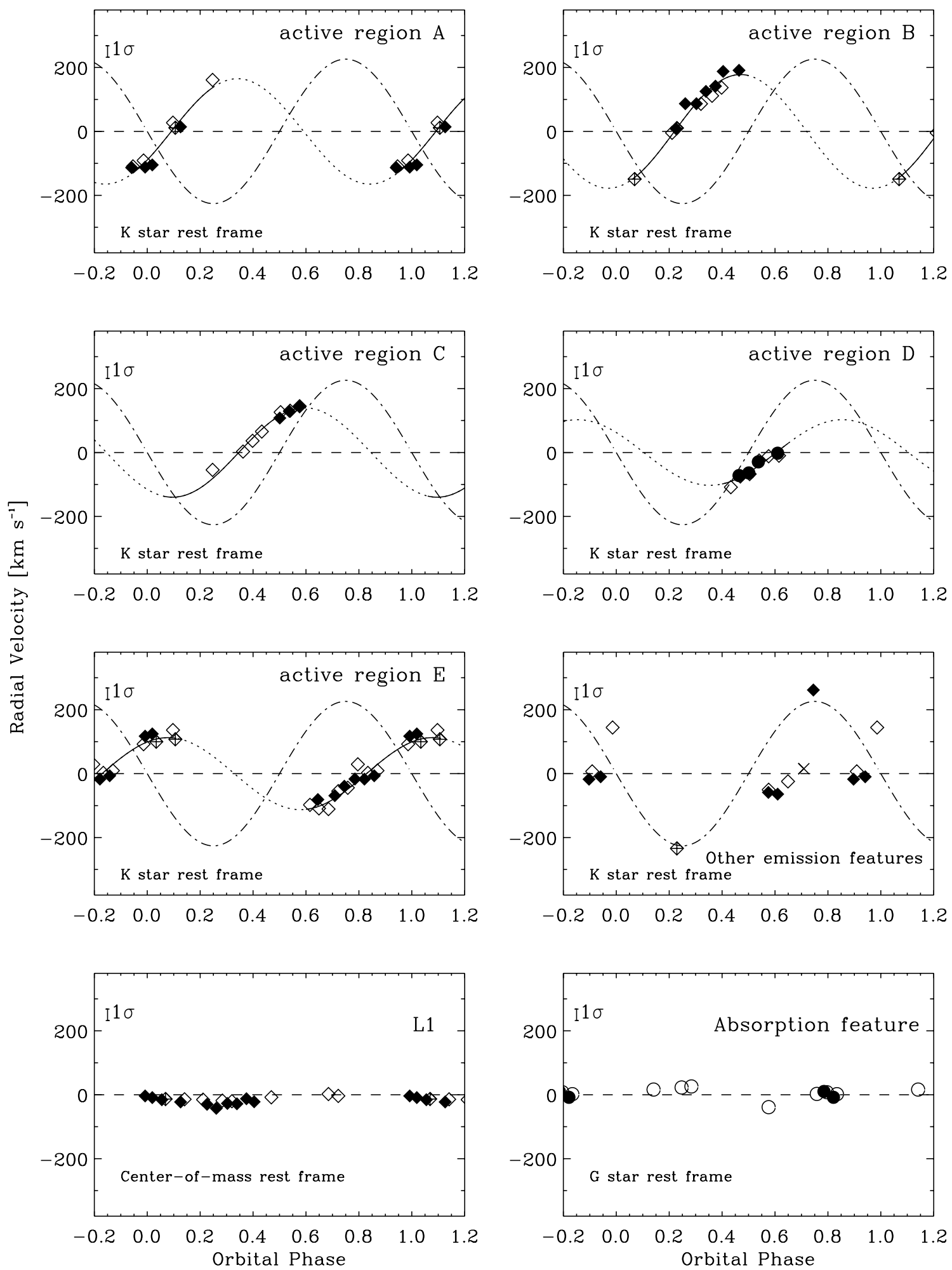

Fig. 8. Radial velocities versus orbital phase of the features that overlap the quiet stellar emission, found by multi-Gaussian fits to the line profiles. Open, filled, and crossed-in symbols are used for the 1st, 2nd and 3rd stellar orbits, respectively. In the 5 panels starting from the top we plot the radial velocities of five active regions versus phase in the K0 IV star rest frame. In thebottom-left panel we show the radial velocity of the $L 1$ emission feature in the center-of-mass rest frame. In the bottom-right panel the radial velocity of an absorption feature detected towards the G2 IV star is plotted versus phase in the G2 IV star rest frame. In the "Other emission features" panel, the cross data point at phase $\sim 0.7$ corresponds to an absorption feature, while all other points represent emission features sporadically detected feature 

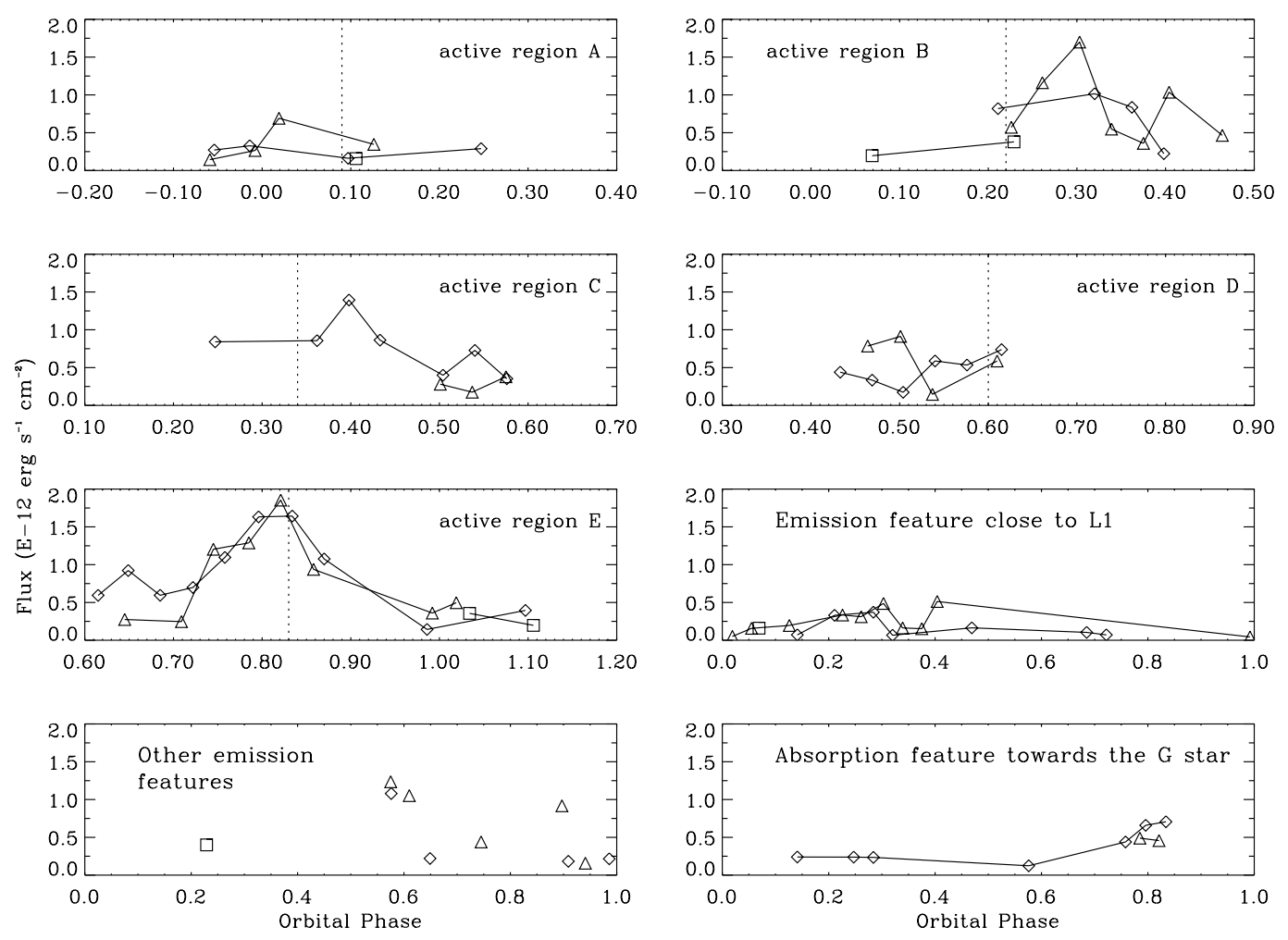

Fig. 9. Integrated fluxes of the additional components as functions of phase. The vertical dotted lines indicate the phase at which the features interpreted as active regions cross the central meridian. Diamond symbols are used for the 1st orbit, triangles for the 2nd orbit, and squares for the 3th one

occupied by the active region. The active region parameters are listed in Table 6.

The active regions $B, C$, and $D$ were detected mainly on only one wing of the K0 IV star line profile. This is likely due to our fitting the profiles with a maximum of five Gaussian components and the overlap of fainter active regions by brighter active regions at similar radial velocities. For example, (a) the active regions $A$ and $E$ are more easily detectable than the active region $B$ at the phases when the active region $B$ would be on the blue wing of the K0 IV star line profile; (b) the active region $B$ and the region close to the Lagrangian point $L 1$ dominates over the active region $C$ between phases 0.1 and 0.3 ; and (c) the absorption feature in front of the G2 IV star and the active region $E$ are more pronounced on the line profile after the active region $D$ has crossed the central meridian. The third repeated coverage of phases between 0.1 and 0.2 allow us to ascertain that the active regions $A, B$, and $E$ have a minimum life time of $\sim 4$ days. The other two active regions were detected during both of the observed orbits. Thus their life times were at least of 2 days.

The fluxes of the individual active regions and other components as a function of phase are shown in Fig. 9. The flux of the active region $A$ was nearly constant during its visibility. The active region $E$ showed a clear flux enhancement, equal in both of the orbits, at the central meridian passage, when it also had a larger line width.
The mean active region fluxes $(f)$ are given in Table 6 , as well as the standard deviation of the measured values that are representative of the flux variability. We also give the active region mean surface fluxes $(F)$ in the $\mathrm{Mg}$ II $h \& k$ lines computed as:

$F_{\mathrm{pl}}=1.76 f_{\mathrm{pl}} \frac{q^{\mathrm{K}}}{\mu H^{\prime 2}}$

where $q^{\mathrm{K}}=d^{2} / R_{\mathrm{K}}^{2}$, with $d$ the distance and $R_{\mathrm{K}}$ the radius of the K0 IV star and $\mu$ and $H^{\prime}$ are the active region filling factors and heights in units of the stellar radius from the stellar center. The factor 1.76 in Eq. (3) was used to compute the $\mathrm{Mg}$ II $h \& k$ flux from the flux in the $k$ line only, assuming $f_{k} / f_{h}=1.32$ as given in Sect. 3 .

Some of the additional emission components could not be identified with long-lived active regions. Looking at their radial velocities ("Other emission features" panel of Fig. 8), we have observed discrete emission features related to the G2 IV star $(\phi \sim 0.23$, and 0.75$)$ that were probably sporadic brightening, and some other features (all at other phases) related to the K0 IV star.

Figure 10 is a sketch of the AR Lac chromospheric map resulting from the spectral imaging of the $\mathrm{Mg}$ II $k$ line. We have not found any discrete feature on the Mg II $k$ line profile migrating from the blue to red wings with amplitude less than the stellar $v_{\mathrm{e}} \sin i$. We believe that this result is due to the difficulty of separating such possible features from the broad quiet stellar emission without higher 


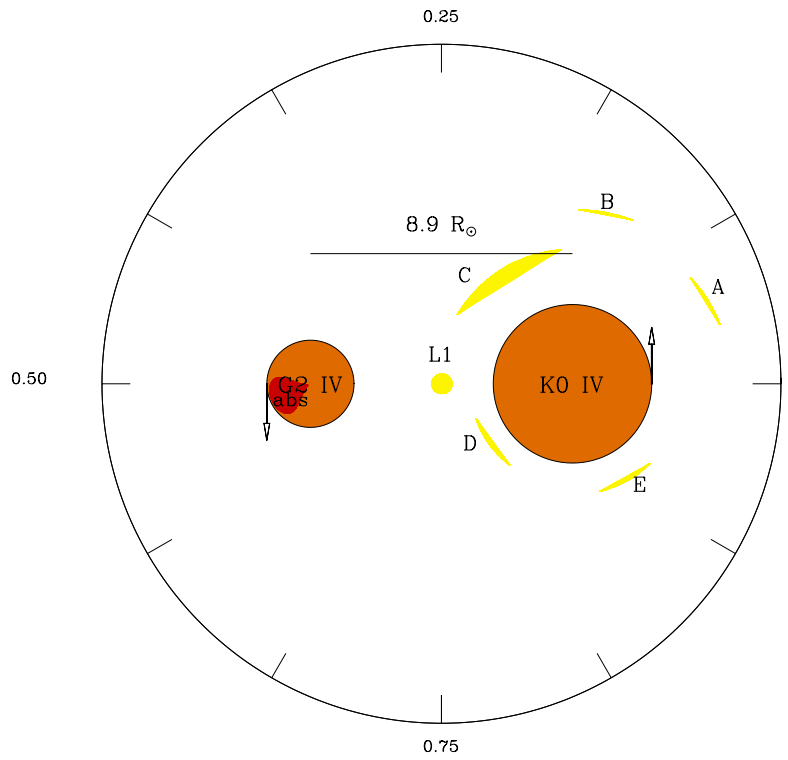

Fig. 10. A sketch of the results of the Mg II spectral imaging of the AR Lac system. The location of the active regions (A through E), of an absorption feature (abs), and of an emission region near the system center of mass (L1) are shown

Table 6. Parameters of the active regions detected on the K0 IV star chromosphere. The last column lists the reduced chi-square values for the sinusoidal curves that best fit the radial velocity data

\begin{tabular}{crrrrrrr}
\hline Name & $L$ & $H_{\min }^{a}$ & $\Delta L^{b}$ & $\mu$ & $f^{c} \pm \sigma^{d}$ & $F^{e} \pm \sigma^{d}$ & $\chi^{2}$ \\
\hline $\mathrm{A}$ & $32^{\circ}$ & 1.0 & $10^{\circ}$ & 0.008 & $2.9 \pm 0.5$ & $7.6 \pm 1.0$ & 1.7 \\
$\mathrm{~B}$ & $79^{\circ}$ & 1.2 & $9^{\circ}$ & 0.006 & $7.2 \pm 1.2$ & $20.9 \pm 2.8$ & 1.0 \\
$\mathrm{C}$ & $122^{\circ}$ & 0.7 & $27^{\circ}$ & 0.054 & $6.3 \pm 1.2$ & $3.3 \pm 0.6$ & 0.5 \\
$\mathrm{D}$ & $216^{\circ}$ & 0.3 & $16^{\circ}$ & 0.019 & $5.2 \pm 0.8$ & $13.7 \pm 1.7$ & 0.5 \\
$\mathrm{E}$ & $299^{\circ}$ & 0.4 & $15^{\circ}$ & 0.017 & $8.0 \pm 1.2$ & $20.2 \pm 2.4$ & 0.9 \\
\hline
\end{tabular}

${ }^{a}$ In units of $R_{\star}$ above the stellar photosphere.

${ }^{b}$ Angular radius upper limit.

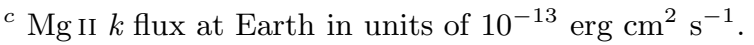

${ }^{d} \sigma$ is 1 standard deviation.

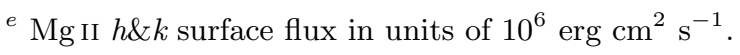

spectral resolution than IUE could provide. In fact, only features that have wide velocity oscillations around the line center are easily detectable and recognized as active regions in the IUE data.

\section{Discussion}

The $\mathrm{Mg}$ II $h \& k$ surface fluxes are $4.08 \pm 0.5210^{6}$ and $6.28 \pm 0.5910^{6} \mathrm{erg} \mathrm{s}^{-1} \mathrm{~cm}^{-2}$ for the $\mathrm{K}$ and $\mathrm{G}$ chromospheric quiet emission, respectively. The reported errors are $\pm 1 \sigma$ (one standard deviation) for all of the measured values. Therefore, in a plot of the Mg II $h \& k$ surface flux as a function of the stellar $B-V$ color (see Fig. 11 adapted from Vilhu 1987), the Mg II $h \& k$ quiet emission from both stars in the AR Lac binary is well below the saturation line in that plot. On the contrary, the Mg II $h \& k$ surface

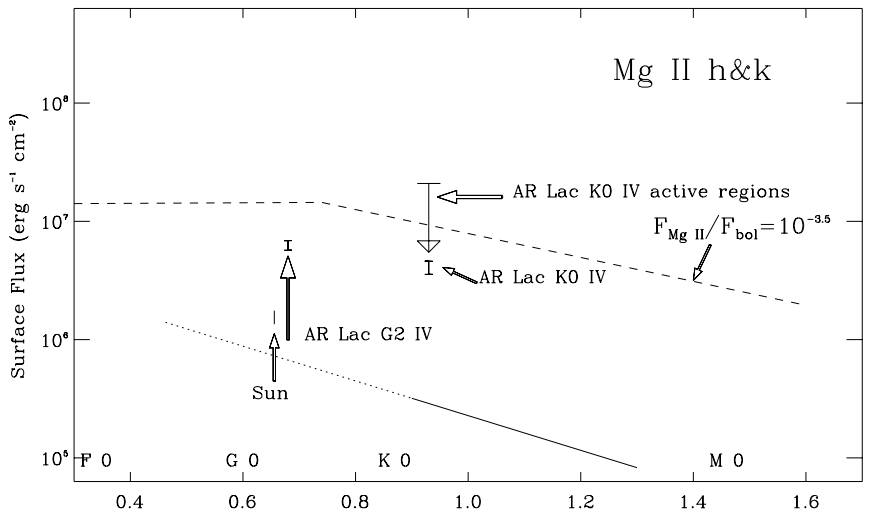

Fig. 11. The $\mathrm{Mg}$ II $h \& k$ surface fluxes of the active regions detected on the $\mathrm{K}$ star and the quiet emission of the $\mathrm{K}$ and $\mathrm{G}$ stars of AR Lac plotted vs. $B-V$ (adapted from Vilhu 1987). The saturation boundary, defined by the fastest rotators (RS CVn binaries, naked T Tau stars and some other young stars), is drawn with a dashed line. The solid line and its extrapolation (dots) represent the $\mathrm{Mg}$ II basal flux according to Schrijver (1987)

fluxes derived for the active regions (see Table 6 ) are close to or exceed the saturation limit $\left(\sim 10^{7} \mathrm{erg} \mathrm{s}^{-1} \mathrm{~cm}^{-2}\right)$. Actually, the exact values of the active region $\mathrm{Mg}$ II $h \& k$ surface fluxes depend on how closely the active region cross section area (for which we estimated upper limits) and height above the photosphere (for which we estimated lower limits) approximate the actual values. Since we do not expect that the height can exceed about $2 R_{\star}$, the $\mathrm{Mg}$ II $h \& k$ surface fluxes of the active regions $C, D$, and $E$ must be at least half of the computed values. If the actual filling factors are lower than estimated, the surface fluxes can be much higher. For example, the computed surface flux of the active region $C$ is lower than the K0 IV star quiet surface flux. This suggests that we have overestimated the dimension of this active region. For an active region radius half the value given in Table 6 , a $\mathrm{Mg}$ II $h \& k$ surface flux 8 times greater would result for the active region $C$. In any case, the mean $\mathrm{Mg}$ II $h \& k$ active region surface flux indicates that the heating mechanism in these active regions operates close to saturation. This result confirms earlier conclusions by Linsky (1991) that the active regions on active stars are regions of saturated heating both in the chromosphere and in the higher temperature regions.

\section{Conclusions}

During the 5 days of observations we detected five flare episodes in the transition region (TR) emission lines. None of these flares was visible in the chromospheric lines. Specifically, the detection of flares in the C II (whose temperature of line formation is $\log T=4.6$ ) but not in the Si II (whose temperature of line formation is $\log T=4.3$ ) emission lines suggests that the physical mechanism responsible for the flares can be effective only in a narrow range of temperatures. 
The AR Lac light curve obtained from chromospheric and TR emission lines shows eclipses that are much wider in phase than predicted by the geometrical eclipses of the stellar photospheres. This fact implies that the plasma at temperature between about 10000 and $200000 \mathrm{~K}$ extends well above the stellar photospheres. Actually, the high resolution Mg II $h \& k$ line profiles show that only the K0 IV star has wide eclipses, while the G2 IV star chromosphere seems to be much more confined.

Multi-Gaussian fits to a sequence of $51 \mathrm{Mg}$ II $k$ line profiles, covering slightly more than 2 stellar orbits, allowed us to describe the properties of the quiet emission from the two stellar chromospheres and to identify localized regions of enhanced chromospheric emission (active regions).

The chromospheric emission of the G2 IV star appears to be both compact and spatially homogeneous. On the other hand, the K0 IV star chromosphere, even apart from the discrete regions that we have identified, is spatially non uniform. Its outer hemisphere (centered at longitude 0) has the weakest Mg II emission.

Some of the regions of enhanced chromospheric emission are corotating with the K0 IV star and are localized at heights between 0.3 and $1.2 R_{\star}$ above the K0 IV star photosphere. Their life-time was greater than our 2-4 days of coverage. The mean Mg II $h \& k$ surface fluxes of these active regions are indicative of a heating mechanism operating in a regime close to saturation.

As discussed by Rodonò et al. (1999), repeated X-ray observations of AR Lac (Walter et al. 1983; White et al. 1990; Ottmann et al. 1993; Siarkowski et al. 1996; Rodonò et al. 1999), as well as EUV observations (Christian et al. 1996) show that even though the spatial structure of the corona of this system is highly variable with time, the $\mathrm{X}$ ray/EUV emission from the G2 IV star typically comes from regions very close to the photosphere, whereas an extended corona surrounds the K0 IV star. Therefore, the scenario indicated by the X-ray observations is consistent with that emerging from our analysis of the $\mathrm{Mg}$ II emission. Moreover, the presence of bright plasma in the $\mathrm{Mg}$ II $h \& k$ line at distances as large as $1.2 R_{\mathrm{K}}$ above the K0 IV star photosphere indicates that multi-temperature structures are cospatial around the K0 IV star.

We have also observed absorbing material in the lines of sight toward both the K0 IV star and the G2 IV star. While the former was a transient event, the latter can be interpreted as due to some obscuring material corotating with the G2 IV star at a latitude greater than 50 degrees. In their analysis of the IUE data set acquired in 1985, Neff et al. (1989) found a pronounced dark spot on the surface of the G2 IV star. This dark spot results from the failure of the Mg II emission from the G2 IV star to reappear as the G2 IV star emerged from eclipse. Although the possibility of a very dark hemisphere cannot be excluded, it now seems plausible that Neff et al. (1989) may have seen an absorption event, in which the G2 IV star is located behind a large extended cool structure, analogous to what is inferred from the 1994 data set presented in this paper. More direct evidence for the existence of cool structures, that lag behind the K0 IV star, comes from the EUV light curve. In particular, Walter (1996a) found optically thick obscuring material confined within $15^{\circ}$ of the equator of the K0 IV star and extending outward by $\sim R_{\mathrm{K}}$ from the light curve of AR Lac observed with the EUVE DS photometer $(70-190 \AA)$. Cool extended structures were also observed on the active single early $\mathrm{K}$ dwarf AB Dor in $\mathrm{H} \alpha$ (Collier-Cameron et al. 1990) and in UV emission lines (Walter 1996b). Extended absorbing structures were also seen in the UV emission lines from the eclipsing K2 V-dA binary V471 Tau by Walter \& Kim (2000).

We find a bright $\mathrm{Mg}$ II feature close to the L1 Lagrangian point, which is probably a signature of some hot spot due to mass exchange between the two stars. Evidence for a similar intersystem structure was found for the RS CVn-type binary system HR 1099 by Busà et al. (1999) and, independently, by Richards \& Rosolowsky (1998).

Finally, we find that the mean central wavelength of the ISM absorption feature in the Mg II $k$ line toward AR Lac is $2796.3354 \pm 0.0094 \AA\left(-1.8 \pm 1.0 \mathrm{~km} \mathrm{~s}^{-1}\right)$ according to the analysis of the 1994 data set. This value was refined to $-1.0 \pm 0.4 \mathrm{~km} \mathrm{~s}^{-1}$ by analyzing 221 of the $240 \mathrm{LWP}(/ \mathrm{R})$ high resolution spectra of AR Lac acquired by IUE since 1979 .

Acknowledgements. Research on stellar activity at Catania University and Astrophysical Observatory is supported by MURST (Ministero dell'Università e della Ricerca Scientifica e Tecnologica). Research at JILA is supported by NASA grant S-56500-D. Research at SUNY was supported in part by NASA ADP grant NAG52983. ZsK is grateful to the Hungarian OTKA Office for grants T-026165, T-032846 and to the Hungarian Space Office for the grant TP096/2000. We wish to thank the referee Dr. N. Griffiths for helpful comments and suggestions.

\section{References}

Ayres, T. R. 1980, The Universe at Ultraviolet Wavelengths, in The first two years of International Ultraviolet Explorer, ed. R. D. Chapman, NASA CP 2171, 237

Barylak, M., \& Ponz, J. D. 1998, ASP Conf. Ser. 145, ed. R. Albrecht, R. N. Hook, \& H. A. Bushouse, 404

Bevington, P. R. 1969, in Data Reduction and Error Analysis for the Physical Science (New York: McGraw-Hill)

Boggess, A., et al. 1978, Nat, 275, 372

Busà, I., Pagano, I., Rodonò, M., Neff, J. E., \& Lanzafame, A. C. 1999, A\&A, 350, 571

Bielicz, E., Glebocki, R., \& Sikorski, J. 1985, A\&A, 153, 269

Cassatella, A., Altamore, A., González-Riestra, R., et al. 2000, A\&AS, 141, 331

Collier-Cameron, A., Duncan, D. K., Ehrenfreund, P., et al. 1990, MNRAS, 247, 415

Christian, D. J., Drake, J. J., Patterer, R. J., Vedder, P. W., \& Bowyer, S. 1996, AJ, 112, 751

Elgarøy, Ø., Engvold, O., \& Jorås, P. 1997, A\&A, 326, 165

Garhart, M. P., Smith, M. A., Tunrose, B. E., Levay, K. L., \& Thompson, R. W. 1997, IUE NEWSIPS Information Manual (Ver. 2.0), NASA IUE Newsletter \#57 
Kővári, Zs., Pagano, I., Neff, J. E., Rodonò, M., \& Walter, F. M. 2000, in Cool Stars, Stellar Systems and the Sun, ed. M. R. Zapatero Osorio, R. García López, \& R. Rebolo, in press

Lanza, A. F., Catalano, S., Cutispoto, G., Pagano, I., \& Rodonò, M. 1998, A\&A, 332, 541

Linsky, J. L. 1991, in Mechanisms of Chromospheric and Coronal Heating, ed. P. Ulmschneider, E. R. Priest, \& R. Rosner (Berlin: Springer-Verlag), 166

Marino, G., Catalano, S., Frasca, A., \& Marilli, E. 1998, IBVS, 4599

Naftilan, S. A., \& Drake, S. A. 1977, ApJ, 216, 508

Neff, J. E., Walter, F. M., Rodonò, M., \& Linsky, J. L. 1989, A\&A, 215, 79

Nichols, J. S., \& Linsky, J. L. 1996, AJ, 111, 517

Ottmann, R., Schmitt, J. H. M. M., \& Kürster, M. 1993, ApJ, 413, 710

Özeren, F. F., Doyle, J. G., \& Jevremovic, D. 1999, A\&A, 350, 635

Pagano, I. 1993, Ph.D. Thesis, Univ. of Catania

Pagano, I., Rodonò, M., \& Neff, J. E. 1992a, Lec. Notes Phys. 397, ed. P. B. Byrne, \& D. J. Mullan, 315

Pagano, I., Rodonò, M., \& Neff, J. E. 1992b, ASP Conf. Ser. 26, ed. M. Giampapa, \& J. Bookbinder, 362

Pagano, I., Rodonò, M., Neff, J. E., \& Walter, F. M. 1994, ASP Conf. Ser. 64, ed. J. P. Caillault, 450

Pagano, I., Rodonò, M., Neff, J. E., \& Walter, F. M. 1995, IAU Coll. 153, ed. Y. Uchida, T. Kosugi, \& H. S. Hudson, 631
Perryman, M. A. C., \& the HYPPARCOS Science Team 1997, ESA SP-1200, vols. 1-12, ESA Pubblication division, c/o ESTEC, Noordwijk, The Netherlands

Richards, M. T., \& Rosolowsky, E. W. 1998, ASP Conf. Ser. 154, ed. R. A. Donahue, \& J. A. Bookbinder, CD-2038

Rodonò, M., Pagano, I., Leto, G., et al. 1999, A\&A, 346, 811

Rodriguez Pascual, P. M. 1993, ESA IUE Newsletter, \#42, 8

Sanford, R. F. 1951, ApJ, 113, 299

Siarkowski, M., Pres, P., Drake, S. A., White, N. E., \& Singh, K. P. 1996, ApJ, 473, 470

Schrijver, C. J. 1987, Lect. Notes Phys., 291, 135

Strassmeier, K. G., Hall, D. S., Fekel, F. C., \& Scheck, M. 1993, A\&AS, 100, 173

Thompson, R. W., Bradley, R. E., \& Teays, T. J. 1990, NASA IUE Newsletter, \#42, 63

Vilhu, O. 1987, Lect. Notes Phys., 291, 110

Walter, F. M. 1996a, IAU Coll. 152, ed. S. Bowyer \& R. F. Malina, 129

Walter, F. M. 1996b, IAU Symp., 176, 355

Walter, F. M., Gibson, D. M., \& Basri, G. S. 1983, ApJ, 267, 665

Walter, F. M., Neff, J. E., Gibson, D. M., Linsky, J. L., \& Rodonò, M. 1987, A\&A, 186, 241

Walter, F. M., \& Kim, S. 2000, in Cool Stars, Stellar Systems and the Sun, ed. M. R. Zapatero Osorio, R. García López, \& R. Rebolo, in press

White, N. E., Shafer, R. A., Horne, K., Parmar, A. N., \& Culhane, J. L. 1990, ApJ, 350, 776 